OPEN ACCESS

Edited by:

Alina Maria Holban,

University of Bucharest, Romania

Reviewed by:

Stuart Friess,

Washington University in St. Louis,

United States

Pia M. Vidal,

Catholic University of the Most Holy

Conception, Chile

*Correspondence:

Xiao-jie Lu

xiaojieluwuxi@163.com

Qi Wu

nswuqi@163.com

Specialty section:

This article was submitted to Microbial Immunology,

a section of the journal

Frontiers in Immunology

Received: 24 October 2021 Accepted: 09 December 2021 Published: 24 December 2021

Citation:

Yuan B, Lu X-j and Wu Q (2021) Gut Microbiota and Acute Central Nervous System Injury: A New Target for

Therapeutic Intervention.

Front. Immunol. 12:800796. doi: 10.3389/fimmu.2021.800796

\section{Gut Microbiota and Acute Central Nervous System Injury: A New Target for Therapeutic Intervention}

\author{
Bin Yuan ${ }^{1}$, Xiao-jie $L u^{1,2 *}$ and Qi Wu ${ }^{3 *}$ \\ ${ }^{1}$ Department of Neurosurgery, The Affiliated Wuxi No. 2 Hospital of Nanjing Medical University, Wuxi, China, ${ }^{2}$ Department of \\ Neurosurgery, The Affiliated Hospital of Jiangnan University, Wuxi, China, ${ }^{3}$ Department of Neurosurgery, Jinling Hospital, \\ Nanjing University, School of Medicine, Nanjing, China
}

Acute central nervous system (CNS) injuries, including stroke, traumatic brain injury (TBI), and spinal cord injury (SCI), are the common causes of death or lifelong disabilities. Research into the role of the gut microbiota in modulating CNS function has been rapidly increasing in the past few decades, particularly in animal models. Growing preclinical and clinical evidence suggests that gut microbiota is involved in the modulation of multiple cellular and molecular mechanisms fundamental to the progression of acute CNS injuryinduced pathophysiological processes. The altered composition of gut microbiota after acute CNS injury damages the equilibrium of the bidirectional gut-brain axis, aggravating secondary brain injury, cognitive impairments, and motor dysfunctions, which leads to poor prognosis by triggering pro-inflammatory responses in both peripheral circulation and CNS. This review summarizes the studies concerning gut microbiota and acute CNS injuries. Experimental models identify a bidirectional communication between the gut and CNS in post-injury gut dysbiosis, intestinal lymphatic tissue-mediated neuroinflammation, and bacterial-metabolite-associated neurotransmission. Additionally, fecal microbiota transplantation, probiotics, and prebiotics manipulating the gut microbiota can be used as effective therapeutic agents to alleviate secondary brain injury and facilitate functional outcomes. The role of gut microbiota in acute CNS injury would be an exciting frontier in clinical and experimental medicine.

\section{Keywords: gut microbiota, stroke, traumatic brain injury, spinal cord injury, gut-brain axis}

\section{INTRODUCTION}

Acute injuries to the central nervous system (CNS), such as stroke, traumatic brain injury (TBI), spinal cord injury (SCI), are critical global health problems that result in lifelong disabilities or death, leading to catastrophic changes to the injured individuals, alongside their family and even the entire community $(1,2)$. The processes secondary to acute CNS injuries involve a sequence of complex pathophysiological mechanisms, including excitotoxicity, electrolyte imbalance, oxidative stress, inflammation, apoptosis, pyroptosis, ferroptosis, autophagy, and cerebral edema (3). These cellular and molecular damages exacerbate neuronal cell death. Although some preclinical researchers have made many efforts to develop efficacious treatment strategies, patients with 
severe acute CNS injuries still have a poor prognosis. Given the prevalence of acute CNS injury-induced disabilities or death, exploring a novel and effective therapeutic regimen is imperative. In addition, a growing body of studies has shown that gut microbiota plays a pivotal role in health and disease in the host, particularly in the CNS (4-7).

Gut microbiota refers to the assemblage of bacteria, archaea, viruses, and eukaryotic microbes that colonize in the digestive tract (8). The gut microbiota contains trillions of microorganisms, over 1000 different species of known bacteria, and approximately $100 \sim 150$-fold more genes than the human genome (9). At the phylum level, the gut microbiota primarily consists of Firmicutes, Bacteroidetes, Actinobacteria, Proteobacteria, and Verrucomicrobia (10). Thereinto, Firmicutes, and Bacteroidetes comprise about $90 \%$ of all the bacteria (11). Additionally, the composition of gut microbiota among individuals was influenced by diet, age, gender, environment, and genes (12). Although the microbiome is spatially restricted to the gut, it has been shown to regulate the functions of distant organs (13). Notably, advances in the sequencing of gut microbiota have revealed the close correlation between the complex ecosystem and CNS (14). Previous studies focused on exploring the bidirectional communication pathways between gut microbiota and CNS, termed the "microbiota-gut-brain" axis (MGBA) (15). The bidirectional communication pathways between the CNS and gut microbiota involve immunological, endocrine, metabolic, and neural pathways $(16,17)$. Recent findings have implicated that MGBA partakes in the pathogenesis of many neurological disorders such as neurodegenerative diseases(e.g., Alzheimer's disease and Parkinson's disease), neurodevelopmental and neuropsychiatric diseases (e.g., anxiety, depression, autism, and schizophrenia), autoimmune disease (e.g., multiple sclerosis), and acute CNS injuries (e.g., stroke, TBI and SCI) $(7,15,18-$ 20). In this review, we provide an update on the link between gut microbiota and acute CNS injuries.

\section{MICROBIOTA-GUT-BRAIN AXIS}

\section{Top-Down Signaling: Brain-to-Gut}

In top-down signaling, the pathways involve the autonomic nervous system, enteric nervous system, the hypothalamicpituitary-adrenal (HPA) axis, and immunological pathway (Figure 1). The autonomic nervous system regulates intestinal homeostasis, and the neurotransmitters, released by the activated sympathetic and parasympathetic nerve fibers, modulate gut motility, gut barrier permeability, fluid maintenance, bile secretion, resident immune cell activation, and gut microbiota makeup (21). The enteric nervous system is also responsible for gut functions, such as gut motility and fluid maintenance, a neuronal connection between the microbiota and the host. Moreover, the HPA axis is one of the vital nonneuronal transmission pathways within the MGBA, releasing cortisol to influence gut homeostasis in response to various stimuli. With the discovery of meningeal lymphatic vessels, the brain is no longer an immune-privileged site. Functional meningeal lymphatic vessels lined mainly in the dorsal part of the skull are responsible for the clearance of cerebrospinal fluid and drainage of immune cells and the periphery, communicating with the host organs (22).

\section{Bottom-Up Signaling: Gut-to-Brain}

Two different mechanisms involved in the bottom-up signaling are neuronal and nonneuronal pathways (Figure 1). The vagus nerve, composed of both afferent and efferent fibers (80\% vs. $20 \%$ ), plays a pivotal role in bidirectionally transmitting vital information between the gut and brain. The afferent fibers stimulated by microbial metabolites and enteroendocrine neuropeptides convey hypothalamic neurons that promote pituitary secretions. In addition, the interaction between the gut and brain primarily relies on the nonneuronal pathway. Singh et al. reported that the gut microbiota-mediated neuroprotection was absent in lymphocyte-deficient mice after an experimental stroke of permanent distal middle cerebral artery occlusion (MCAO), indicating that the gut communicate with the brain by immunological pathway (23). In a transient MCAO model, intestinal CD $45^{+}$and $\mathrm{CD} 11 \mathrm{c}^{+}$cells significantly migrated from the gut to the brain and meninges at 3 days poststroke (24). A study of a thoracic level 9 contusion SCI also showed that commercial probiotics (VSL\#3) feeding triggered a protective immune response in gut-associated lymphatic tissues (GALTs) and conferred neuroprotection with the improvement of locomotor recovery after SCI (25). TBI-induced leaky gut released lipopolysaccharide (LPS), a toxic bacterial component, into the circulation that exacerbated neuroinflammation by activating microglia (26).

The MGBA is critical to the development of the human central nervous system. A prospective longitudinal study conducted by Carlson et al. investigated the correlation between gut microbial composition and cognitive ability in 89 infants. It revealed that 2-year-old cognitive function assessed with the Early Learning Composite of Mullen Scale was significantly correlated with the gut microbiota composition at one year (27). Infants with a relatively high abundance of Bacteroides showed higher performance, while those with a relatively high quantity of Faecalibacterium had a lower performance. In another clinical study of 39 infants, the $\alpha$ diversity of gut microbiota was associated with functional connectivity between the amygdala and thalamus, between the anterior cingulate cortex and anterior insula, and between the supplementary motor area and the inferior parietal lobule (28). Additionally, the functional connectivity was also related to 2year-old cognitive outcomes. These studies have demonstrated that the gut microbiota significantly affects neurodevelopment in the early stage through the MGBA.

Microbial components and metabolites such as lipopolysaccharide (LPS), long-chain fatty acids (LCFAs), short-chain fatty acids (SCFAs), trimethylamine-N-oxide (TAMO), tryptophan, and polysaccharide A (PSA) are considered to induce neuroinflammation and modulate the function of CNS either directly or by activating migration of peripheral immune cells to the brain. Although this regulation of 


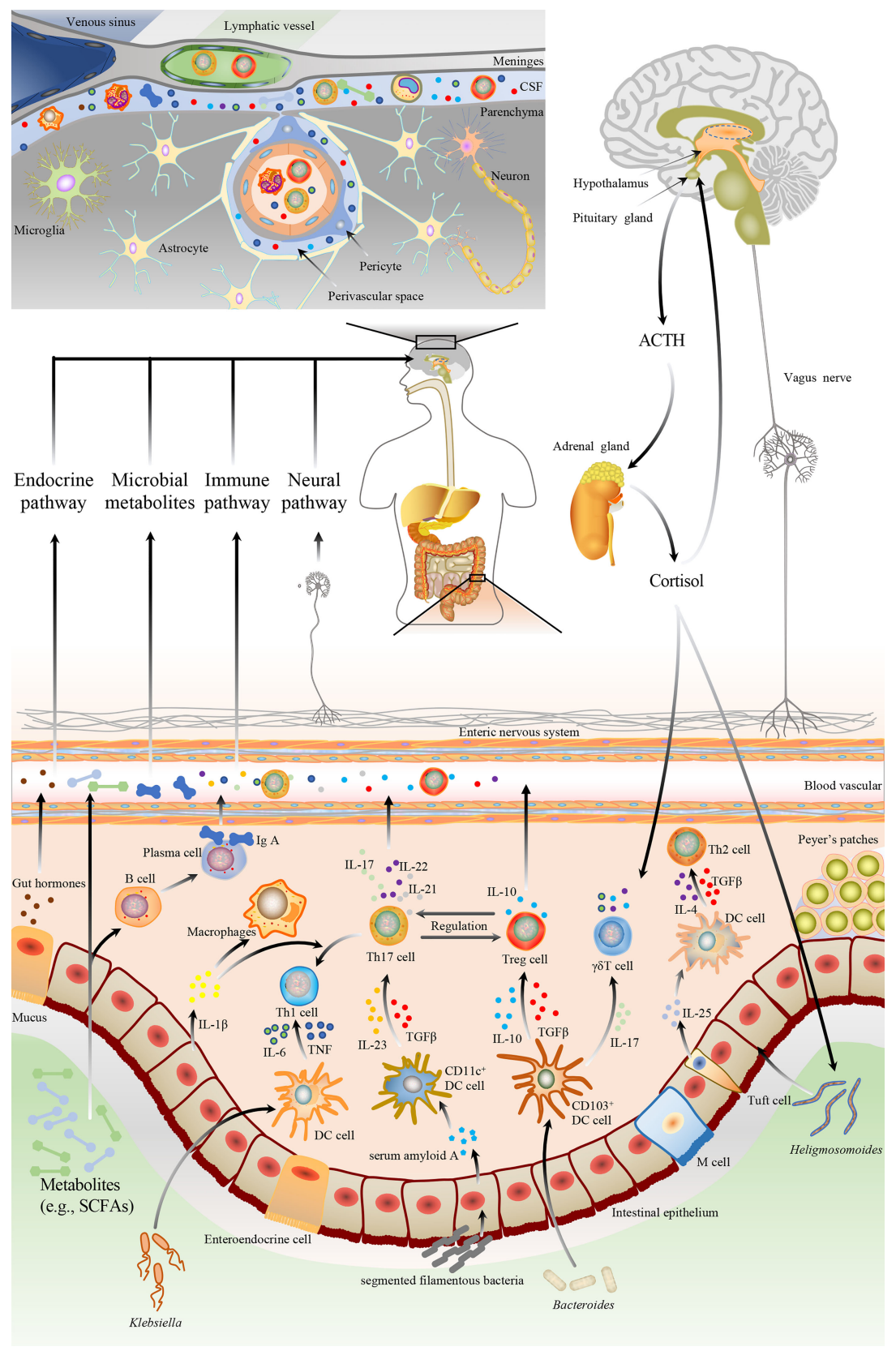

FIGURE 1 | The bidirectional communication pathways between the gut microbiota and brain. The gut microbiota could bi-directionally communicate with the brain through multiple pathways, including neuronal and non-neuronal. The brain regulates the gut microbiota via neuronal pathways (e.g., autonomic nervous system and enteric nervous system), hypothalamic-pituitary-adrenal axis, etc. Neuronal pathways release neurotransmitters to modulate gut motility, gut barrier permeability, fluid maintenance, resident immune cell activation, and gut microbiota composition. HPA also releases cortisol to regulate gut homeostasis. Additionally, gut microbiota affects the development and pathophysiology of the brain by immunological, endocrine, metabolic, and neural pathways. Microbiomes and their metabolites could modulate the brain and behavior by affecting intestinal epithelial cells to alter gut barrier function, enteroendocrine cells to secret hormones, as well as dendritic cells and macrophage, to regulate immune and microglia activation. Gut microbiota can modulate the $\mathrm{CD} 4^{+} \mathrm{T}$ cells differentiation through epithelial cells or DC cellsmediated signals. (1) Ectopic colonizing microbes, such as Klebsiella, can invade intestinal epithelium and stimulate DC cells to secrete proinflammatory cytokines, including IL-6 and TNF, which drive the polarization of Th1cells. (2) SFB promotes Th17 polarization via epithelial cell-mediated CD11 ${ }^{+}$DC cells activation. Epithelial cells release serum amyloid A to activate CD11 ${ }^{+}$DC cells, leading to the TGF- $\beta$, IL-12, and IL-23 secretion. (3) Resident microbes, such as Bacteroides, modulate Treg cells generation by TGF- $\beta$ and IL-10, which are secreted by CD103 ${ }^{+}$DC cells. CD103 ${ }^{+}$DC cells also can release IL-17 to promote $\gamma \delta$ T cell polarization. (4) Microbiota-associated Th2 cell polarization is correlated with parasite colonization such as Heligmosomoides, mediated by tuft cells secreting IL-25 to DC cells. Then activated DC cells release IL-4 and TGF- $\beta$ to drive Th2 polarization. DC, dendritic cell; IL, interleukin; TNF, tumor necrosis factor; Th, T helper; TGF- $\beta$, transforming growth factor- $\beta$; SFB, segmented filamentous bacteria; SCFA, short-chain fatty acid; Treg, regulatory $T$ cell. 
immune cells by the microbiota occurs in the gut, peripheral immune cells could also migrate to the brain meninges and modulate the brain function (29).

\section{GUT MICROBIOTA AND IMMUNOMODULATION}

\section{Gut Microbiota}

Previous studies have shown that the gut microbiota-host interaction contributes to the maturation and modulation of the host immune system. Through constant contact with the gut microbiota, immune cells and epithelial cells located in the gut have achieved a homeostatic state and promote tolerogenic responses to the host commensal microbes. Under physiological conditions, all types of immune cells such as $\mathrm{T}$ lymphocytes, B lymphocytes, macrophages, dendritic cells (DCs), etc., counterbalance each other to preserve the host homeostasis. $\mathrm{T}$ helper (Th) cells and regulatory $\mathrm{T}$ (Treg) cells are a requisite component of the host immune system, especially in the gut-associated immune responses. Compared with germfree (GF) mice, conventional specific-pathogen-free (SPF) mice had a more significant proportion of $\mathrm{CD}^{+}{ }^{+} \mathrm{T}$ cells (30). Th1 cells were polarized by DCs-secreted pro-inflammatory cytokines such as interleukin(IL)-6 and IL-12 stimulated by Klebsiella (31). Parasites, such as Heligmosomoides, could activate Th2 cells through DCs-derived transforming growth factor- $\beta$ (TGF$\beta$ ) and IL-4 (32). Besides, segmented filamentous bacteria drive Th17 polarization via activation of $\mathrm{CD}_{11 \mathrm{c}^{+}} \mathrm{DCs}$ (33). The activated Th17 cells secrete high-affinity IL-17 and promote IgA transportation, memory $\mathrm{CD}^{+} \mathrm{T}$ cell differentiation, and mucin production (33). Bacteroides fragilis stimulate regulatory $\mathrm{CD}^{+} \mathrm{T}$ cells to make themselves colonize the intestinal epithelium and induce immunosuppression, mediated by $\mathrm{CD}_{103^{+}} \mathrm{DCs}^{(34)} \gamma \delta \delta \mathrm{T}$ cells, another innate immune cell population in the gut epithelium, are vital for gut homeostasis regulation and pathological reaction. $\mathrm{CD}_{103}{ }^{+}$DCs activated by gut microbiota also communicate with IL- $17^{+} \gamma \delta$ T cells via cellto-cell contact or different cytokines (35). $\gamma \delta \mathrm{T}$ cells protect the host from intestinal barrier damage and pathogenic bacterial invasion and exert pro-inflammatory or anti-inflammatory effects depending on the milieu (36). Additionally, the development of intestinal mucosa B cells is regulated by commensal microbes, which promote antibody production and control the expression of a differentiation-related gene through enhancement of fatty acid synthesis, glycolysis, and oxidative phosphorylation (37). Commensal microbiota-induced secretion of IL- $1 \beta$ by mucosal macrophages is closely correlated with the development of steady-state Th17 cells (38)(Figure 1).

\section{Microbial Components and Metabolites}

Gut microbiota-derived small molecules are inextricably linked with the crosstalk between gut microbiota and host. Moreover, there is now a considerable body of experimental evidence that some metabolites of the intestinal microbiota can participate in the modulation of inflammatory cytokine production and immune cell differentiation. SCFAs produced by gut microbiota such as Faecalibacterium prausnitzii, Roseburia intestinalis, and Anaerostipes butyraticus regulate the activation and differentiation of immune cells (e.g., neutrophils, macrophages, DCs, and T cells), mediated by inhibiting histone deacetylases (HDACs) as well as activating G-protein-coupled receptors (GPCRs) (e.g., GPR41, GPR43, and GPR109A) and olfactory receptor-78 (Olfr-78) (39-41). TMAO is another gut microbiota metabolite oxidized from trimethylamine (TMA) generated by hepatic flavin-containing monooxygenases (FMOs) from dietary nutrients such as choline and L-carnitine. TAMO regulates pro-inflammatory responses via activating the NOD-like receptor family, pyrin domaincontaining protein 3 (NLRP3) inflammasome, mitogenactivated protein kinase (MAPK), and nuclear factor- $\mathrm{\kappa B}$ (NF$\kappa \mathrm{B})$ signaling pathway (42). Additionally, tryptophan has also been demonstrated to be involved in the functional modulation of intestinal intraepithelial lymphocytes and innate lymphocytes via activating the aryl hydrocarbon receptor (AHR) (43). PSA, produced by Bacteroides fragilis, activates toll-like receptor 2 (TLR2) expressed on DCs and Treg cells, triggering antiinflammatory cytokine IL-10 (43). PSA also regulates the differentiation of naive $\mathrm{CD}^{+} \mathrm{T}$ cells into Th1 cells, skewing Th1/Th2 ratio towards Th1 cells (44). Interestingly, a study found that CD39 expression is required for Treg cells to migrate to the CNS, depending on PSA-driven effects on the Treg cells (45).

\section{GUT MICROBIOTA AND ACUTE CNS INJURIES}

\section{Stroke}

Stroke is the second leading cause of death worldwide. Morbidity and mortality of stroke grow in many countries, contributing to financial burden and loss of life quality, and thus diminishing national happiness index. Approximately 15 million people around the world are victims of a stroke every year (46). There are two types of strokes: ischemic stroke and hemorrhagic stroke. Recent evidence shows that shifts in the gut microbial composition occur rapidly after stroke in clinical and experimental studies. Therefore, alteration of the gut microbiota directly regulates the reactions secondary to brain injury through central and peripheral immunity and indirectly determines the brain's sequel to these types of catastrophic events.

\section{Ischemic Stroke}

Reportedly, ischemic stroke accounts for $\sim 80 \%$ of all strokes (47), and the gut microbiota plays an essential role in the pathogenesis and prognosis of ischemic stroke. Multiple studies have shown that ischemic stroke significantly changes the gut microbiota composition $(21,23,48-66)$. These studies have been summarized in Table 1. Although dysbiosis of the gut microbiota has been proved in previous studies, controversy still exists on the specific microbiota difference. Compelling evidence has been identified that confounding factors such as 
TABLE 1 | A summary of preclinical and human studies on the gut microbiota and ischemic stroke.

\begin{tabular}{|c|c|c|c|}
\hline & Subjects & Methods & Key findings \\
\hline $\begin{array}{l}\text { Yin J, } \\
\text { et al. } \\
(2015) \\
(48)\end{array}$ & 322 patients vs. 231 controls & $\begin{array}{l}16 \mathrm{~S} \text { rRNA (V4) } \\
\text { sequencing \& LC-MS }\end{array}$ & $\begin{array}{l}\text { - Patients with stroke and transient ischemic attack presented the gut microbiota dysbiosis, which } \\
\text { increased Enterobacter, Megasphaera, Oscillibacter, and Desulfovibrio and decreased } \\
\text { Bacteroides, Prevotella, and Faecalibacterium. } \\
\text { - Patients with stroke and the transient ischemic attack had lower trimethylamine N-oxide (TMAO) } \\
\text { compared with asymptomatic patients. }\end{array}$ \\
\hline $\begin{array}{l}\text { Stanley D, } \\
\text { et al. } \\
\text { (2016) }\end{array}$ & $\begin{array}{l}36 \text { patients vs. } 9 \text { hospital-based controls } \\
\text { vs. } 10 \text { healthy controls; } \\
\text { middle cerebral artery occlusion (MCAO) }\end{array}$ & 16S rRNA sequencing & $\begin{array}{l}\text { - Common commensal bacteria resided in the intestinal tracts contributed to the post-stroke } \\
\text { infections in patients with ischemic stroke. This was also observed in a mouse model of ischemic } \\
\text { stroke. }\end{array}$ \\
\hline (52) & mice & & $\begin{array}{l}\text { - In the experimental stroke, post-stroke infection was only seen in specific pathogen-free (SPF) } \\
\text { mice, not germ-free (GF) mice. }\end{array}$ \\
\hline $\begin{array}{l}\text { Nie J, } \\
\text { et al. }\end{array}$ & 622 patients vs. 622 controls & LC-MS & $\begin{array}{l}\text { - } \quad \text { The increment of serum TMAO level increased the risk of the first stroke. } \\
\text { - } \quad \text { Patients with higher TMAO levels }(\geq 1.79 \mu \mathrm{mol} / \mathrm{L}) \text { had a significantly higher risk of the first stroke. }\end{array}$ \\
\hline
\end{tabular}

(51)

Zeng $\mathrm{X}, \quad 141$ patients

et al.

16S rRNA sequencing \& GS-MS

Compared with the low-risk group, opportunistic pathogens (e.g., Enterobacteriaceae and (2019) Veillonellaceae) and lactate-producing bacteria (e.g., Bifidobacterium and Lactobacillus) were increased, as well as butyrate-producing bacteria (e.g., Lachnospiraceae and Ruminococcaceae) were decreased in the high-risk group.

(50)

- The fecal butyrate concentrations in the high-risk group were lower than those in the low-risk group. Moreover, the concentrations of other short-chain fatty acids (SCFAs) (e.g., acetate, propionate, isobutyrate, isovalerate, and valerate) in the feces were significantly different between the three groups.

Haak BW, 349 patients vs. 51 controls

et al.

(2020)

(49)

Xu DJ,

et al.

(2021)

(60)

Ling $Y$,

et al.

(2020)

(61)

Xiang L, 20 patients vs. 16 controls

et al.

(2020)

(62)

Tan C, 140 patients vs. 92 controls

et al.

(2021)

(67) asymptomatic controls (PSNCl)
61 large artery atherosclerotic (LAA) stroke vs. 20 cardioembolic (CE) stroke vs. 51

53 patients with post-stroke cognitive impairment ( $\mathrm{PSCl}$ ) vs. 40 patients with post-stroke non-cognitive impairment 16S rRNA (V4)
sequencing \& GS-MS

16S rRNA (V3-V4) sequencing

16S rRNA (V3-V4) sequencing \& LC-MS

16S rRNA (V4-V5) sequencing \& LC-MS

- The TMAO level in stroke patients was two-fold lower than that of the healthy controls.

- Lower abundance of butyrate-producing bacteria within $24 \mathrm{~h}$ of hospital admission was an independent predictor of enhanced risk of post-stroke infection, but not of mortality or functional patient outcome.

- The TMAO levels in the plasma of patients with LAA and CE strokes were significantly higher than those in controls. Moreover, the plasma TMAO level in the LAA stroke patients was positively associated with the carotid plaque area.

- The composition and the function of gut microbiota in the patients with LAA stroke were significantly different from those in the asymptomatic controls. In contrast, no significant difference between CE stroke patients and the asymptomatic controls was observed in the present study.

- Compared with the patient with PSNCl, the abundance of Proteobacteria was highly increased in the patients with PSCl.

- The abundances of Clostridia, Clostridiales, Lachnospiraceae, and Lachnospiraceae_other were significantly decreased in the patients with PSCl after adjusting to age.

- The Kyoto Encyclopedia of Genes and Genomes analysis showed the progressive enriched module for folding, sorting, and degradation (chaperones and folding catalysts) and the significantly decreased modules related to metabolisms of cofactors and vitamins, amino acid, and lipid in patients with PSCl.

$16 \mathrm{~S}$ rRNA (V3) - Stroke patients had fewer Firmicutes than controls.

sequencing

- Two optimal bacterial species, Lachnospiraceae (OTU_45) and Bacteroides served as markers of lacunar infarction.

- Two optimal bacterial species, Bilophila and Lachnospiraceae (OTU_338)), served as markers of non-lacunar acute ischemic infarction.

- Three optimal bacterial species, Pseudomonas, Sphingomonadaceae, and Akkermansia, served as markers of post-ischemic stroke patients with 15 days of treatment.

- Patients with acute ischemic stroke are characterized by a lack of SCFAs-producing bacteria (Roseburia, Bacteroides, Lachnospiraceae, Faecalibacterium, Blautia, and Anaerostipes) and an overload of Lactobacillaceae, Akkermansia, Enterobacteriaceae, and Porphyromonadaceae in their feces.

- The SCFAs levels were negatively related to stroke severity and prognosis.

- Reduced fecal SCFAs level, especially acetate, was correlated with an increased risk of 3-month unfavorable outcomes.

Zhang J, 351 patients vs. 150 controls $\quad$ LC-MS

et al.

(2021)

(68)

Guo Q, $\quad 49$ patients vs. 30 controls

et al.
16S rRNA (V3-V4) sequencing
- Patients with an unfavorable outcome had significantly increased plasma TMAO levels on admission.

- Plasma TMAO levels on admission were an independent predictor of functional outcome and mortality after acute ischemic stroke.

- The acute ischemic stroke patients treated with Tanhuo Decoction had a better outcome than the controls on both clinical outcome and gut microbiota characteristics. 
TABLE 1 | Continued

Subjects Methods

(69)

Huang $Y, \quad 76$ patients vs. 19 controls

et al.

(2021)

(63)

\begin{tabular}{|c|c|}
\hline $\begin{array}{l}\text { Sun T, } \\
\text { et al. } \\
\text { (2021) } \\
(70)\end{array}$ & 953 patients vs. 953 controls \\
\hline Xu K, & Cohort 1: 28 patients vs. 28 controls; \\
\hline et al. & Cohort 2: 124 patients; \\
\hline 021) & MCAO mice \\
\hline
\end{tabular}

(64)

\begin{tabular}{|c|c|c|}
\hline $\begin{array}{l}\text { Houlden } \\
\text { A, et al. } \\
(2016) \\
(21)\end{array}$ & MCAO mice & $16 \mathrm{~S}$ rRNA PCR \\
\hline $\begin{array}{l}\text { Singh V, } \\
\text { et al. } \\
(2016) \\
(54)\end{array}$ & MCAO mice & $\begin{array}{l}\text { 16S rRNA (V1-V3) } \\
\text { sequencing }\end{array}$ \\
\hline $\begin{array}{l}\text { Benakis } \\
\text { C, et al. } \\
(2016) \\
(55)\end{array}$ & MCAO mice & $\begin{array}{l}16 \mathrm{~S} \text { rRNA (V4-V5) } \\
\text { sequencing }\end{array}$ \\
\hline $\begin{array}{l}\text { Winek K, } \\
\text { et al. } \\
(2016) \\
(59)\end{array}$ & MCAO mice & - \\
\hline $\begin{array}{l}\text { Spychala } \\
\text { MS, et al. } \\
(2018) \\
(53)\end{array}$ & MCAO mice & $\begin{array}{l}16 \mathrm{~S} \text { rRNA (V4-V5) } \\
\text { sequencing }\end{array}$ \\
\hline $\begin{array}{l}\text { Singh V, } \\
\text { et al. } \\
(2018) \\
(23)\end{array}$ & MCAO mice & $\begin{array}{l}\text { 16S rRNA (V1-V3) } \\
\text { sequencing }\end{array}$ \\
\hline $\begin{array}{l}\text { Benakis } \\
\text { C, et al. } \\
(2020) \\
(56)\end{array}$ & MCAO mice & $\begin{array}{l}\text { 16S rRNA (V4) } \\
\text { sequencing }\end{array}$ \\
\hline
\end{tabular}
sequencing

LC-MS/MS
$16 \mathrm{~S}$ rRNA (V3-V4) - Stroke patients had a significantly higher abundance of Enterococcus and lower abundances of

$16 S$ rRNA sequencing

- Tanhuo Decoction treatment significantly decreased the lipopolysaccharide (LPS)-producing bacteria (Bacteroidaceae and Bacteroides) to reduce LPS biosynthesis.

- The acute ischemic stroke patients treated with Tanhuo Decoction also exhibited the potential to decrease the biosynthesis of trimethylamine (TMA), the precursor of TMAO, and increase TMA's degradation.

Bacteroides, Escherichia-Shigella, and Megamonas.

- Compared with stroke patients, patients with post-stroke cognitive impairment had a significantly higher proportion of Enterococcus, Bacteroides, and Escherichia-Shigella and a lower content of Faecalibacterium.

- Patients with the post-stroke affective disorder had a significantly higher proportion of Bacteroides and Escherichia-Shigella and a lower proportion of Enterococcus and Faecalibacterium.

- Plasma TMAO levels in patients with ischemic stroke were significantly increased.

- Higher plasma TMAO levels were correlated with increased odds of ischemic stroke.

- The adjusted odds ratios for ischemic stroke per $1 \mu \mathrm{mol} / \mathrm{L}$ increase of plasma TMAO was 1.05.

- Enriched Enterobacteriaceae was an independent risk factor for acute ischaemic stroke patients in early-stage recovery.

- MCAO mice showed rapid gut dysbiosis with Enterobacteriaceae blooming, associated with intestinal ischemia and nitrate production.

- Enterobacteriaceae exacerbates brain infarction by accelerating LPS/toll-like receptor 4(TLR4)mediated systemic inflammation.

- Inhibiting Enterobacteriaceae overgrowth by diminishing nitrate generation or inhibiting nitrate respiration alleviates brain infarction.

- The alteration of the caecal microbiota composition following stroke could be mediated by noradrenaline release from the autonomic nervous system, changing caecal mucoprotein production and goblet cell numbers.

- Specific changes in Peptococcaceae and Prevotellaceae after stroke were correlated with the severity of the injury.

- Reduced species diversity and bacterial overgrowth of bacteroidetes were associated with intestinal barrier dysfunction and reduced intestinal motility.

- GF mice recolonized with poststroke gut microbiota exacerbates infarct volume and functional deficits following stroke, mediated by the migration of intestinal pro-inflammatory $T$ cells to the ischemic brain.

- Fecal microbiota transplantation (FMT) could normalize brain lesion-induced dysbiosis and improve stroke outcomes.

- Antibiotic-induced alterations in the gut microbiota reduced brain injury after ischemic stroke.

- Dysbiosis following ischemic stroke changed intestinal immune homeostasis, leading to an increase in regulatory $\mathrm{T}(\mathrm{Treg})$ cells and a reduction in IL-17+ $\gamma \delta \mathrm{T}$ cells through altered dendritic cell activity. Moreover, dysbiosis blocked the migration of effector $\mathrm{T}$ cells from the gut to the leptomeninges.

- Microbiota-depleted mice stopped the antibiotic cocktail pretreatment 3 days before surgery significantly decreased survival after MCAO.

- Microbiota-depleted animals treated by continuous antibiotic treatment or colonized with SPF microbiota before surgery rescued from severe acute colitis.

- The Firmicutes to Bacteroidetes ratio in aged mice increased $~ 9$-fold compared to young.

- The gut microbiota in the young manipulated by fecal from aged mice increased mortality, decreased behavioral performance, and increased cytokine levels following MCAO, altering the microbiota in the aged by fecal gavage to resemble that of young increased survival and improved recovery following MCAO.

- Bacterial colonization reduces stroke volumes by increasing cerebral expression of cytokines and microglia/macrophage cell counts.

- The gut microbiota-mediated neuroprotection was absent in lymphocyte-deficient mice.

- Single antibiotic treatment with either ampicillin or vancomycin, but not neomycin, significantly reduced the infarct volume and improved motor sensory function 3 days after stroke.

- Bacteroidetes S24.7 and the enzymatic pathway for aromatic metabolism were correlated with infarct size.

- The gut microbiota composition in the ampicillin-treated mice was associated with reduced gut inflammation, a long-term favorable outcome, and a reduction of brain tissue loss.

- Regulation of SCFAs and tryptophan pathways induced by ampicillin could be predictive of stroke outcomes. 
TABLE 1 | Continued

\begin{tabular}{|c|c|c|c|}
\hline & Subjects & Methods & Key findings \\
\hline $\begin{array}{l}\text { Sadler R, } \\
\text { et al. } \\
(2020) \\
(57)\end{array}$ & MCAO and photothrombotic (PT) mice & GC-MS & $\begin{array}{l}\text { - SCFAs supplementation in the drinking water significantly improved recovery of limb motor } \\
\text { function by altering contralesional cortex connectivity, which is related to SCFAs-dependent } \\
\text { changes in spine and synapse densities. } \\
\text { - A substantial impact of SCFAs on microglial activation contributes to the structural and functional } \\
\text { remodeling, mediated by the recruitment of T cells to the infarcted brain. }\end{array}$ \\
\hline $\begin{array}{l}\text { Lee J, } \\
\text { et al. } \\
(2020) \\
(58)\end{array}$ & MCAO mice & $\begin{array}{l}16 \mathrm{~S} \text { rRNA (V4) } \\
\text { sequencing \& LC-MS }\end{array}$ & $\begin{array}{l}\text { - Aged stroke mice transplanted the young fecal improved post-stroke neurological deficits and } \\
\text { inflammation, which correlated with higher SCFAs levels and SCFAs-producers such as } \\
\text { Bifidobacterium longum, Clostridium symbiosum, Faecalibacterium prausnitzii, and Lactobacillus } \\
\text { fermentum. }\end{array}$ \\
\hline $\begin{array}{l}\text { Jeon J, } \\
\text { et al. } \\
(2020) \\
(65)\end{array}$ & MCAO pig & $\begin{array}{l}\text { 16S rRNA (V3-V4) } \\
\text { sequencing }\end{array}$ & $\begin{array}{l}\text { - Compared with pre-stroke populations, the abundance of the Proteobacteria was significantly } \\
\text { increased, while the abundances of Firmicutes and lactic acid bacteria Lactobacillus decreased } \\
\text { at } 3 \text { days poststroke. } \\
\text { - The gut microbial pattern returned to similar values as prestrike at } 5 \text { days poststroke. }\end{array}$ \\
\hline $\begin{array}{l}\text { Benakis } \\
\text { C, et al. } \\
(2020) \\
(56)\end{array}$ & MCAO mice & $\begin{array}{l}\text { 16S rRNA (V4) } \\
\text { sequencing }\end{array}$ & $\begin{array}{l}\text { - Mice treated with a cocktail of antibiotics significantly reduced infarct volume in the acute phase } \\
\text { of stroke. } \\
\text { - Single antibiotic treatment with either ampicillin or vancomycin, but not neomycin, significantly } \\
\text { reduced infarct volume and improved neurological function } 3 \text { days after stroke. } \\
\text { Bacteroidetes S24.7 and the enzymatic pathway for aromatic metabolism were associated with } \\
\text { infarct size after stroke. } \\
\text { - The gut microbiota signature in the ampicillin-treated mice was correlated with reduced intestinal } \\
\text { inflammation, long-term favorable outcome and was predictive of SCFAs and tryptophan } \\
\text { pathways. }\end{array}$ \\
\hline $\begin{array}{l}\text { Huang Q, } \\
\text { et al. } \\
(2021) \\
(71)\end{array}$ & MCAO rat & $\begin{array}{l}\text { 16S rRNA (V3-V4) } \\
\text { sequencing \& GC-MS }\end{array}$ & $\begin{array}{l}\text { - Compared with non-hemorrhagic transformation }(\mathrm{HT}) \text { rats, the relative abundances of } \\
\text { - } \quad \text { Total SCFAs levels, especially butyrate and valeric acid, were significantly decreased in the cecal } \\
\text { contents of HT rats. } \\
\text { - The rats colonized with gut microbiota from HT rats showed increased susceptibility to HT. }\end{array}$ \\
\hline $\begin{array}{l}\text { Zhang P, } \\
\text { et al. } \\
\text { (2021) } \\
\text { (72) }\end{array}$ & MCAO mice & $\begin{array}{l}16 \mathrm{~S} \text { rRNA sequencing } \\
\& \text { HPLC-MS }\end{array}$ & $\begin{array}{l}\text { - Atorvastatin significantly ameliorated neurological defects and reduced microglia-mediated } \\
\text { neuroinflammation after experimental stroke. } \\
\text { - Atorvastatin increased the abundance of Firmicutes and Lactobacillus, decreased Bacteroidetes } \\
\text { abundance, increased fecal butyrate level, promoted intestinal barrier function by elevating the } \\
\text { expression of claudin-1, occludin and mucoprotein 2, as well as regulated intestinal immune } \\
\text { function. } \\
\text { - Transplantation of atorvastatin-treated mice fecal microbiota alleviated neuroinflammation in } \\
\text { MCAO mice. }\end{array}$ \\
\hline $\begin{array}{l}\text { Huang JT, } \\
\text { et al. } \\
(2021) \\
(73)\end{array}$ & MCAO mice & $16 \mathrm{~S}$ rRNA sequencing & $\begin{array}{l}\text { - The transplantation of gut microbiota collected from calorie-restriction-treated mice was eligible } \\
\text { to have better long-term rehabilitation. } \\
\text { - } \quad \text { Bifidobacterium was enriched in calorie-restriction mice. } \\
\text { - Bifidobacterium administration improved the long-term rehabilitation of stroke mice }\end{array}$ \\
\hline $\begin{array}{l}\text { Zhu W, } \\
\text { et al. } \\
(2021) \\
(74)\end{array}$ & MCAO mice & $\begin{array}{l}16 \mathrm{~S} \text { rRNA (V4) } \\
\text { sequencing \& LC-MS }\end{array}$ & $\begin{array}{l}\text { - The human fecal microbial transplantation study showed TMAO production and stroke severity } \\
\text { - TMAO and choline supplementation exacerbated infarct size and functional impairment. } \\
\text { - Gut microbial CutC increased host TMAO levels and aggravated cerebral infarct size and } \\
\text { functional deficits after stroke. }\end{array}$ \\
\hline $\begin{array}{l}\text { Wu W, } \\
\text { et al. } \\
(2021) \\
(66)\end{array}$ & MACO rat & $\begin{array}{l}\text { 16S rRNA (V3-V4) } \\
\text { sequencing \& LC-MS }\end{array}$ & $\begin{array}{l}\text { - The abundance of the Firmicutes phylum was decreased, whereas Proteobacteria and } \\
\text { Deferribacteres were increased after stroke. } \\
\text { - } \quad \text { Ruminococcus_sp_15975 might serve as a biomarker for the stroke. } \\
\text { - Many metabolites, such as L-leucine, L-valine, and L-phenylalanine, differed between the stroke } \\
\text { and sham groups, mainly involved in mineral absorption and cholinergic synapse pathways. }\end{array}$ \\
\hline $\begin{array}{l}\text { Yuan Q, } \\
\text { et al. } \\
(2021) \\
(75)\end{array}$ & MCAO mice & $\begin{array}{l}\text { 16S rRNA sequencing } \\
\& \text { GC-MS }\end{array}$ & $\begin{array}{l}\text { - Lactulose supplementation significantly improved the functional outcome after stroke by } \\
\text { downregulating inflammatory reaction and increased anti-inflammatory factors in the brain and } \\
\text { gut. } \\
\text { - Lactulose supplementation improved intestinal barrier injury and restored gut microbiota } \\
\text { dysbiosis after stroke. }\end{array}$ \\
\hline
\end{tabular}

16 rRNA, 16 S ribosomal RNA.

$P C R$, polymerase chain reaction.

LC-MS, liquid chromatography-mass spectrometry.

GC-MS, gas chromatography-mass spectrometry.

HPLC-MS, high-performance liquid chromatography-mass spectrometry. 
age, diet, behavior, antibiotic use, prolonged stress, environment, and genetics compose the gut microbiota, which may be influenced by the contradictory results of the above studies. Thus, more studies are needed to clarify the role of gut microbiota dysbiosis in the pathogenesis and prognosis of ischemic stroke. Recently, a preclinical study also suggested that the alteration in the gut microbiota was associated with hemorrhagic transformation (HT) (71). The relative abundance of Proteobacteria and Actinobacteria was significantly increased in HT rats after experimental stroke, indicating that the gut microbiota is involved in the progression of ischemic stroke.

Mechanistically, the gut microbiota-mediated neuroprotection greatly depended on the microglia and lymphocyte responses, significantly increasing Th cells, polarized Treg cells, and Th17 cell counts in the intestinal Peyer's patches (23). Proinflammatory Th1, Th17, and $\gamma \delta \mathrm{T}$ cells often increase inflammatory damage, while Treg cells suppress post-stroke inflammation by secreting the anti-inflammatory cytokine IL10. Alteration of gut microbiota following a stroke in the bacterial population triggers pro-inflammatory $\mathrm{T}$ cells responses, migrates intestinal immune cells to the meninges involved in secondary brain injury, and worsens stroke outcome. In GF MCAO animal models, mice transplanted with post-stroke fecal content presented increased infarct volume and functional deficits by inducing pro-inflammatory $\mathrm{T}$ cell polarization. Moreover, restoration of gut microbiota homeostasis with fecal microbiota transplantation (FMT) reduced infarct volume, improved stroke outcome and promoted the migration of intestinal Treg cells to the ischemic area in the brain (54). Also, intestinal dysbiosis following ischemic stroke was found to regulate immune homeostasis in the small intestine with increased Treg cells and decreased IL-17 $\gamma \delta \mathrm{T}$ cells, mediated by DCs. The neuroprotective effect of IL-10 was identified as a regulator of Treg cell-mediated IL- $17^{+} \gamma \delta \mathrm{T}$ cell suppression (55).

Microbial-derived metabolites also correlate with the progression and prognosis of ischemic stroke. Ischemic stroke patients had significant gut microbiota dysbiosis with an increased abundance of SCFAs-producing bacteria such as Odoribacter, Akkermansia, which closely correlated with the stroke outcome (76). However, Zeng et al. reported that people with a high risk of stroke had lower levels of butyrate-producing bacteria (e.g., Lachnospiraceae and Ruminococcaceae) and fecal butyrate (50). Tan et al. also reported a lack of SCFAs-producing bacteria and a low fecal SCFAs level in acute ischemic stroke patients (67). Moreover, the reduced fecal SCFAs were correlated with an increased risk of 3-month unfavorable outcomes (67). The differences in the results of these clinical studies may be due to the small cohorts of the studies. These findings need to be further validated by higher-quality clinical studies with large cohorts. In experimental stroke, reduced plasma SCFAs level correlated with a worse stroke outcome in mice, and SCFAs supplementation improved behavioral recovery with modified poststroke cortical connectivity and synaptic plasticity by recruiting $\mathrm{T}$ lymphocytes on modulation of microglial activation, as reflected by the increase in Treg cells (57). Oral gavage of SCFAs-producing bacteria such as Bifidobacterium longum, Clostridium symbiosum, Faecalibacterium prausnitzii, and Lactobacillus fermentum alleviated post-stroke neurological deficits and inflammation by increasing populations of Treg cells and reducing the percentage of $\mathrm{IL}-17^{+} \gamma \delta \mathrm{T}$ cells (58). Pretreatment with Clostridium butyricum improved neurological deficits and decreased hippocampal apoptosis by increasing butyrate and reducing brain oxidative stress in experimental stroke (77). Furthermore, Zhou et al. reported that butyrate alleviated neuronal apoptosis following stroke via

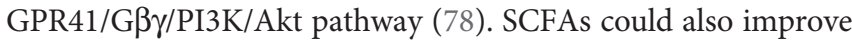
outcomes by protecting gut epithelial cells against strokeinduced gut leakiness by enhancing tight junction proteins (79). Furthermore, sodium butyrate reduced infarct volume and improved neurological function Recently, Huang et al. found that the significant decrease of SCFAs in cecal contents, especially butyrate and valeric acid, was closely related hemorrhagic transformation after ischemic stroke (71).

It has been demonstrated that there is a significant association between TMAO level and various diseases, including stroke (80). Although several clinical studies have identified a correlation between TMAO level and stroke, the results remain controversial. Most studies show that the plasma TMAO concentrations in stroke patients are significantly higher than those in control patients, and its high level is positively related to the severity of the stroke $(51,68)$. A large-scale case-control study with 953 sex- and age-matched pairs performed by Sun et al. suggested that the plasma TMAO concentrations in patients with first acute ischemic stroke were significantly elevated (70). Furthermore, further analysis revealed that the multivariable-adjusted odds ratios for ischemic stroke per 1 $\mu \mathrm{mol} / \mathrm{L}$ increase of plasma TMAO level were 1.05. Tan et al. reported that TMAO concentrations decreased with time after stroke, and elevated TMAO levels at an early stage predicted poor stroke outcomes (81). A meta-analysis also showed that compared with non-stroke controls, TMAO increased the stroke risks by $68 \%$ and accreted $2.201 \mathrm{umol} / \mathrm{L}$ on the average level of TMAO in stroke patients (82). Zhu et al.'s study also suggested gut microbiota can impact stroke severity via the gut microbial CutC-mediated TMAO pathway, which exacerbated cerebral infarct size and functional deficits (74).

The activation of the kynurenine pathway for tryptophan degradation correlates with stroke-induced inflammatory responses and unfavorable outcomes ${ }^{53}$. Tryptophan catabolites regulate intestinal immune cell function by activating AHR. Pharmacological and genetic inhibition of neural cell-specific AHR activation improved stroke outcomes in the MCAO mice model (83). Furthermore, tryptophan catabolism positively correlated with the severity of stroke outcome and might be associated with stroke-induced inflammatory response (51). Besides, xenobiotic/aromatic compound metabolism was a predictive marker of the size of the ischemic lesion (56).

Multiple studies have demonstrated that antibiotic-induced dysbiosis promotes the proliferation and differentiation of $\mathrm{T}$ cells in the gut to either improve or worsen outcomes in experimental stroke. Mice pretreated with ampicillin or vancomycin 
significantly improved the outcome of stroke, whose neuroprotection is related to a shift with increased Proteobacteria and Firmicutes and reduction of Bacteroidetes caused by antibiotics (56). Particularly, Bacteroidetes S24.7 was closely associated with infarct size. However, Winek et al. reported stroke mice pretreated with quintuple broad-spectrum antibiotics presented with the damaged gut epithelium and worsened outcome (59). This controversial result still needs further study to clarify. FMT is a novel and potent treatment strategy in patients with gut microbiota dysbiosis obtained from fecal microbiota in healthy individuals. FMT exerts a neuroprotective effect by altering gut microbial metabolites production and reducing pro-inflammatory gut bacteria, alleviating inflammatory response and oxidative stress in the brain. Restoring gut microbiota homeostasis with FMT from healthy donors reduced lesion size by increasing Treg cells (54). MCAO mice receiving FMT from anti-inflammatory donors reduced the infarct volume by $54 \%$ (55). Additionally, FMT from young microbiota was also beneficial to stroke recovery (53). Oral gavage of SCFAs-producing bacteria or SCFAs supplementation also alleviated neurological deficits and improved poststroke recovery by reducing IL- $17^{+} \gamma \delta \mathrm{T}$ cells in the ischemic brain $(57,58)$. Recently, Zhang et al. found that atorvastatin significantly alleviated the defects in sensorimotor behaviors and reduced microglia-mediated neuroinflammation by increasing the abundance of Firmicutes and Lactobacillus, decreasing the abundance of Bacteroidetes abundance, increasing fecal butyrate level, promoting intestinal barrier function, as well as regulating intestinal immune function (reduced monocyte chemotactic protein 1(MCP-1), tumor necrosis factor- $\alpha($ TNF- $\alpha)$ and increased IL-10) in the mice with permanent MCAO (72). Calorie restriction also can promote ischemic stroke rehabilitation via enriching the abundance of Bifidobacterium (73). Tanhuo decoction also promoted poststroke recovery by decreasing the biosynthesis of TMA, the precursor of TMAO, and increasing the expression of trimethylamine-corrinoid protein Co-methyltransferase ( $\mathrm{mttB})$, which catabolizes TMA to methane (69). Additionally, Lactulose supplementation was shown to significantly improve the functional outcome of stroke, which is possibly mediated by repairing intestinal barrier injury and improving gut microbiota dysbiosis following stroke (75).

\section{Hemorrhagic Stroke}

Hemorrhagic stroke includes intracranial and subarachnoid hemorrhage. Intracranial hemorrhage accounts for $80 \%$ of hemorrhagic stroke and $10-15 \%$ of all strokes, which is primarily caused by hypertension-induced small vessel rupture, while subarachnoid bleeding is mainly due to intracranial aneurysms rupture (84). Hemorrhagic stroke is characterized by high mortality and morbidity, which burdens society and families. However, there are, to date, few studies focused on the exploration of the correlation between hemorrhagic stroke and gut microbiota. A few studies reported that gut microbiota dysbiosis contributes to hypertension and intracranial aneurysms. However, the direct relationship between gut microbiota and hemorrhagic stroke has not been studied. Both clinical and animal studies are warranted in the future.

\section{Gut Microbiota and Intracranial Aneurysms}

A case-control metagenome-wide association study showed that the structural heterogeneity of intestinal microbiota in patients with intracranial aneurysm (IA) was significantly decreased compared to healthy controls, which had an increased abundance of Bacteroides, Parabacteroides, Ruminococcus, and Blautia in IA patients and an enrichment of Faecalibacterium, Eubacterium, Collinsella, and Lactobacillus (85). Recently, another multicenter, prospective case-control study reported that the abundance of the genus Campylobacter and Campylobacter ureolyticus was significantly higher in patients with ruptured IA than that in patients with unruptured IA, which may be associated with the rupture of IA (86). Further analysis suggested that gut microbiota promoted the pathogenesis of IA by regulating plasma amino acids (e.g., taurine, hypotaurine, L-histidine, and L-citrulline) and fatty acid levels (85). Compared to mice transplanted with healthy control feces, the incidence of IA in mice transplanted with the feces of IA patients was significantly increased (85). Mechanistically, supplementation with taurine or H.hathewayi reduces the formation and rupture of IA by blunting cerebrovascular inflammatory processes, reducing extracellular matrix remodeling, and maintaining the structural integrity of cerebral blood vessels. Similarly, Fumiaki Shikata et al. also reported that the gut microbiota contributes to the development of IA by modulating inflammation in the experimental IA model (87). Gut microbiota depletion by an oral antibiotic cocktail of ampicillin, metronidazole, neomycin, and vancomycin (AMNV) significantly reduced the incidence of IA via decreasing macrophage infiltration and the expression of pro-inflammatory cytokines such as IL-1 $\beta$ and IL-6 in vascular wells (87). These results suggest that gut microbiota is closely correlated with the development of IA. Additionally, human studies are needed to determine the exact contribution of the gut microbiota to the pathophysiology of IA and aneurysmal subarachnoid hemorrhage in humans.

\section{Traumatic Brain Injury (TBI)}

Traumatic brain injury (TBI) is one of the most common neurological diseases, with an estimated incidence of approximately 50 million people worldwide annually, leading to thousands of deaths and disabilities (88). TBI induces various secondary progressive brain damage contributing to varied functional outcomes. TBI also influences the gut barrier integrity, gut function, and gut microbiota composition (89). In turn, gut microbiota alterations may regulate a proinflammatory response following TBI and aggravate secondary brain injury. However, the information on TBI-induced gut microbiota dysbiosis is scarce for now. The relevant studies are summarized in Table 2.

Recently, an observational study investigated the characteristics of gut microbiota in $101 \mathrm{TBI}$ patients and found 
TABLE 2 | A summary of preclinical and human studies on the gut microbiota and traumatic brain injury.

\begin{tabular}{|c|c|c|c|}
\hline & Subjects & Methods & Key findings \\
\hline $\begin{array}{l}\text { Mahajan C, } \\
\text { et al. (2021) } \\
\text { (90) }\end{array}$ & 101 patients & - & $\begin{array}{l}\text { - All organisms belonged to the Proteobacteria phylum, especially Enterobacteriaceae forming the largest } \\
\text { group after traumatic brain injury (TBI). } \\
\text { - } \quad \mathrm{TBI} \text { is associated with widespread colonization with Proteobacteria as early as } 48 \text { hours after injury. }\end{array}$ \\
\hline $\begin{array}{l}\text { Hou Y, et al. } \\
(2021)(91)\end{array}$ & $\begin{array}{l}24 \text { patients vs. } 10 \\
\text { controls; surgical } \\
\text { brain injury (SBI) rat }\end{array}$ & $\begin{array}{l}16 S \text { rRNA } \\
\text { sequencing \& } \\
\text { HPLC-MS }\end{array}$ & $\begin{array}{l}\text { - The abundances of Enterococcus, Parabacteroides, Akkermansia, and Lachnoclostridium were significantly } \\
\text { increased, whereas the relative abundances of Bifidobacterium and Faecalibacterium were decreased in the } \\
\text { patients with TBI. } \\
\text { - Oral administration of brain protein combined with probiotics alleviated inflammatory gut damage by } \\
\text { affecting tryptophan-related pathways. }\end{array}$ \\
\hline $\begin{array}{l}\text { Treangen } \\
\text { TJ, et al. } \\
(2018)(92)\end{array}$ & $\begin{array}{l}\text { controlled cortical } \\
\text { impact }(\mathrm{CCl}) \text { mice }\end{array}$ & $\begin{array}{l}\text { 16S rRNA (V3- } \\
\text { V4) sequencing }\end{array}$ & $\begin{array}{l}\text { - At a high-level view, the abundances of Marvinbryantia and Clostridiales were significantly changed after } \\
\text { TBI. } \\
\text { - Lactobacillus gasseri, Ruminococcus flavefaciens, and Eubacterium ventriosum were decreased at the } \\
\text { species level, while Eubacterium sulci and Marvinbryantia formatexigens and were increased after TBI. }\end{array}$ \\
\hline $\begin{array}{l}\text { Li H, et al. } \\
(2018)(93)\end{array}$ & $\begin{array}{l}\text { weight-drop impact } \\
\text { (WDI) mice }\end{array}$ & - & $\begin{array}{l}\text { - Clostridium butyricum treatment improved neurological deficits, brain edema, neurodegeneration, and } \\
\text { blood-brain barrier impairment. } \\
\text { Clostridium butyricum treatment increased tight junction proteins, p-Akt, and Bcl-2 and decreased } \\
\text { expression of Bax. } \\
\text { - Mice treated by Clostridium butyricum showed an increased intestinal Glucagon-like peptide 1(GLP-1) } \\
\text { secretion and upregulated the expression of cerebral GLP-1 receptor. }\end{array}$ \\
\hline $\begin{array}{l}\text { Simon DW, } \\
\text { et al. (2020) } \\
\text { (89) }\end{array}$ & $\mathrm{CCl}$ mice & $\begin{array}{l}16 S \text { rRNA (V4) } \\
\text { sequencing }\end{array}$ & $\begin{array}{l}\text { - Mice receiving pretreatment of ampicillin, metronidazole, neomycin, and vancomycin(AMNV) before surgery } \\
\text { increased CA1's density of hippocampal neuronal and reduced lba-1 positive cells at } 72 \mathrm{~h} \text { after TBI. } \\
\text { - } \quad \text { Mice pretreated by AMNV alleviated associative learning deficit and decreased lesion volume after TBI. }\end{array}$ \\
\hline $\begin{array}{l}\text { Angoa- } \\
\text { Pérez M, } \\
\text { et al. (2020) } \\
\text { (94) }\end{array}$ & WDI mice & $\begin{array}{l}\text { 16S rRNA (V4) } \\
\text { sequencing }\end{array}$ & $\begin{array}{l}\text { - An early increase in microglial activation persisted from 0-day to 90-day post-injury, compounded by } \\
\text { substantial increases in astrocyte reactivity and phosphorylated tau. } \\
\text { - } \quad \text { Few differences in the microbial community were observed in mice exposed to repetitive, mild TBI (rmTBI). } \\
\text { - The progressive emergence of white matter damage and cognitive deficits following rmTBI was not } \\
\text { associated with the altered gut microbiota. }\end{array}$ \\
\hline $\begin{array}{l}\text { Opeyemi } \\
\text { OM, et al. } \\
(2021)(95)\end{array}$ & $\mathrm{CCl}$ mice model & $\begin{array}{l}\text { 16S rRNA (V4) } \\
\text { sequencing \& } \\
\text { HPLC-MS }\end{array}$ & $\begin{array}{l}\text { - Bacteria from Lachnospiraceae, Ruminococcaceae, and Bacteroidaceae families were depleted, while } \\
\text { bacteria from the Verrucomicrobiaceae family were enriched. } \\
\text { - Fecal SCFAs such as acetate were reduced at } 7 \text { days and } 28 \text { days following TBI; SCFAs administration } \\
\text { improved spatial learning after TBI. }\end{array}$ \\
\hline $\begin{array}{l}\text { Du D, et al. } \\
\text { (2021) (96) }\end{array}$ & $\mathrm{CCl}$ rat & $\begin{array}{l}\text { 16S rRNA (V3- } \\
\text { V4) sequencing } \\
\& \text { HPLC-MS }\end{array}$ & $\begin{array}{l}\text { - } \mathrm{TBI} \text { induced significant changes in the gut microbiome, including the alpha- and beta-bacterial diversity and } \\
\text { the microbiome composition at } 8 \text { days after TBI. Fecal microbiota transplantation (FMT) could rescue these } \\
\text { changes and relieve neurological deficits after TBI. } \\
\text { - Metabolomics results showed that the level of trimethylamine (TMA) in feces and the level of trimethylamine } \\
\text { N-oxide (TMAO) in the ipsilateral brain and serum was increased after TBI. At the same time, FMT } \\
\text { decreased TMA levels in the feces and TMAO levels in the ipsilateral brain and serum. } \\
\text { FMT can restore gut microbiota dysbiosis and relieve neurological deficits, possibly through the TMA- } \\
\text { TMAO-methionine sulfoxide reductase A (MsrA) signaling pathway after TBI. }\end{array}$ \\
\hline $\begin{array}{l}\text { You W, } \\
\text { et al. (2021) } \\
(97)\end{array}$ & $\begin{array}{l}\text { lateral fluid } \\
\text { percussion injury } \\
\text { mice model }\end{array}$ & $\begin{array}{l}\text { 16S rRNA (V3- } \\
\text { V4) sequencing } \\
\& \text { HPLC-MS }\end{array}$ & $\begin{array}{l}\text { - The diversity of gut microbiota experienced a time-dependent change from } 1 \mathrm{~h} \text { to } 7 \text { days post-TBI. } \\
\text { - The decreased levels of bile acids, especially secondary bile acids, were related to intestinal inflammation } \\
\text { after TBI. } \\
\text { - Staphylococcus and Lachnospiraceae may contribute to the bile acid metabolic changes. }\end{array}$ \\
\hline $\begin{array}{l}\text { Celorrio M, } \\
\text { et al. (2021) } \\
(98)\end{array}$ & $\mathrm{CCl}$ mice & PCR & $\begin{array}{l}\text { - Antibiotic-induced gut microbial dysbiosis significantly worsened neuronal loss after TBI. } \\
\text { - Antibiotic exposure for } 1 \text { week after TBI decreased T lymphocyte infiltration, increased microglial pro- } \\
\text { inflammatory markers, and reduced cortical infiltration of Ly6C }{ }^{\text {high }} \text { monocytes. } \\
\text { Gut microbiota dysbiosis was associated with increased hippocampal neuronal loss and fear memory } \\
\text { response } 3 \text { months after TBI. }\end{array}$ \\
\hline
\end{tabular}

$16 S$ rRNA, 165 ribosomal RNA.

$P C R$, polymerase chain reaction.

HPLC-MS, high-performance liquid chromatography-mass spectrometry.

that organisms from rectal swabs obtained on days 0,3 , and 7 after admission belonged to the Proteobacteria phylum, with Enterobacteriaceae forming the largest group (90). Hou et al. also analyzed the gut microbiota composition in a small cohort (10 healthy control volunteers vs. 24 TBI patients) and reported that the abundance of Enterococcus, Parabacteroides, Akkermansia, and Lachnoclostridium were significantly increased, while the abundance of Bifidobacterium and Faecalibacterium were decreased in TBI patients (91).

In the controlled cortical impact (CCI) mouse model, the gut microbiota significantly decreased in Lactobacillus gasseri,
Ruminococcus flavefaciens, and Eubacterium ventriosum and increased dramatically in Eubacterium sulci and Marvinbryantia formatexigens at $24 \mathrm{~h}$ post-CCI (92). In an experimental weightdrop injury model, the severity of TBI is correlated with the alteration in Bacteroidetes, Porphyromonadaceae, Firmicutes, and $\alpha$-Proteobacteria (21). Nicholson et al. found a reduced Firmicutes/Bacteroidetes ratio in gut microbiota composition occurring at $2 \mathrm{~h}$ post-injury was significantly related to MRIdetermined lesion volume and behavioral function defects (99). In the lateral fluid percussion injury mice model, You et al. also observed the alterations of gut microbiota and bile acid profile 
(97). Further analysis found that specific bacterial taxa such as Staphylococcus and Lachnospiraceae could be associated with the bile acid metabolic changes, resulting in intestinal inflammation. Interestingly, Angoa-Pérez et al. found that repetitive, mild TBI did not cause alterations in the gut microbiota composition (94). Although differences in gut microbiota composition have been observed after TBI in animal models, the exact regulatory mechanism remains elusive. A study considered that vagal afferent alterations, TBIinduced increase of cholecystokinin level, might be responsible for gut dysfunction through activation of the vago-vagal NTSinhibitory pathway (100). Additionally, in another experimental TBI, the gut upregulated the expression of glycoproteins to recruit immune cells and activate inflammatory signals, resulting in altered mucosal integrity (101). The leaky gut allowed toxic bacterial components such as LPS to enter the circulation that mediates neuroinflammation by activating microglia following TBI (26). Furthermore, the permeability of the blood-brain barrier (BBB) can increase up to 4 times more than normal within $6 \mathrm{~h}$ following TBI. The increased BBB permeability aggravates the gut dysbiosis-induced neuroinflammation by LPS exposure, $\gamma \delta \mathrm{T}$ cell activation, and activated microglia differentiation into the M1 phenotype. A recent preclinical study performed by Celorrio et al. suggested that antibiotic-induced gut microbial dysbiosis established before TBI significantly worsened neuronal loss, reduced cortical infiltration of Ly6 $\mathrm{C}^{\text {high }}$ monocytes and $\mathrm{T}$ lymphocyte, increased microglial pro-inflammatory markers, and impaired neurogenesis after TBI (98).

CCI mice pretreated with AMNV 2 weeks before CCI presented increased neuronal density in the hippocampus at $72 \mathrm{~h}$ post-injury, while mice treated with AMNV right after CCI showed reduced lesion volume and attenuated associative learning deficit at 22 days (89). TBI mice treated by Clostridium butyricum also improved neurological deficits, attenuates brain edema, ameliorated neurodegeneration, and alleviated BBB impairment via elevating intestinal Glucagonlike peptide 1(GLP-1) secretion (93). SCFAs supplementation also improved spatial learning following CCI-induced TBI, mediated by activating the neurotrophic tyrosine kinase receptor type 1 (TrkA) pathway $(95,102)$. Probiotic supplementation also significantly remedied the gut microbiota dysbiosis and decreased the intestinal permeability following experimental TBI by reducing the intestinal mucosa damage, alleviating brain injury (103). In human studies, probiotic treatment could relieve systemic inflammatory response, decrease nosocomial infection rate, and improve the recovery of patients with TBI $(104,105)$. Interestingly, vagal stimulation reduced gut barrier permeability after TBI, mediated by the suppression of TNF- $\alpha$ release (106). Recently, it has been demonstrated that FMT can restore gut microbiota dysbiosis following TBI and ameliorate neurological deficits, mediated by the TMA-TMAO-MsrA signaling pathway (96).

\section{Spinal Cord Injury (SCl)}

Traumatic spinal cord injury (SCI) is another acute CNS injury that affects millions worldwide every year (107). The studies involving SCI and the bidirectional effect on the gut microbiota have been carried out in recent years, which are summarized in Table 3. This section reviews the study published on the changes in the gut microbiota that occur following SCI.

In a Chinese cohort study, Zhang et al. observed an increase in Proteobacteria and Verrucomicrobia and reduced Bacteroidaceae and Bacteroides in patients with chronic traumatic complete SCI (109). Lin et al. also analyzed 46 Chinese subjects (23 SCI patients vs. 23 healthy controls) and reported that the abundances of Parabacteroides, Alistipes, Phascolarctobacterium, Christensenella, Barnesiella, Holdemania, Eggerthella, Intestinimonas, Gordonibacter, Bilophila, Flavonifractor, and Coprobacillus were higher in the patients with SCI than those in the health individuals (110). Another clinical study with 54 Turkish participants (41 SCI patients vs. 13 healthy controls) identified that butyrateproducing microbes of the Firmicutes phylum are significantly reduced in SCI patients than healthy controls (108). Recently, Bazzocchi et al. investigated a large Italian SCI population acute phase after injury and age- and gender-matched healthy Italians (112). Their study revealed that the abundance of SCI patients' gut microbiota increased in potentially pathogenic, proinflammatory, and mucus-degrading bacteria and decreased in SCFAs producers. Moreover, gut microbiome dysbiosis is closely associated with the severity of the lesion after SCI. A case-control study carried by $\mathrm{Yu}$ et al. (45 SCI patients vs. 24 healthy individuals) showed that the abundance of Actinobacteria and Synergistetes in patients with complete thoracic SCI (CTSCI) was significantly higher than that in healthy individuals. At the same time, the Bacteroidetes, Cyanobacteria, and Proteobacteria were significantly decreased in patients with incomplete thoracic SCI (ITSCI) as compared to the healthy (113). Furthermore, they compared the gut microbiota composition between patients with CTSCI and ITSCI and found a significantly increased abundance of Coriobacteriaceae, Synergistetes, Eubacterium, and Cloacibacillus was observed in patients with CTSCI, while patients with ITSCI were abundant with Lactobacillaceae, Lachnospiraceae, Eubacterium, Clostridium, and Sutterella.

Similarly, a thoracic level 9 (T9) contusion SCI-induced gut microbiota dysbiosis in the experimental SCI mice was also characterized by an expansion of Bacteroidetes and a reduction of Firmicutes (115). However, a preclinical work in a T9 contusion SCI mouse model by Kigerl et al. reported that SCI mice presented a decrease in Bacteroidales and an increase in Clostridiales (25). In an SCI rat model, gut microbiota composition was significantly changed with an increased abundance of Lactobacillus intestinalis, Clostridium disporicum, and Bifidobacterium choerinum and a reduced level of Clostridium saccharogumia (114). The difference in the results may be caused by experimental deviation. Additionally, the above analyses of SCI-induced gut microbiota dysbiosis were assessed by $16 \mathrm{~S}$ rRNA amplicon sequencing, which cannot profile microbiota function or identify viruses (123). Du et al. studied gut microbiota dysbiosis after experimental SCI at T4 or T10 using genome- and gene-resolved metagenomic analysis (122). The results suggested that the abundance of beneficial commensals (Lactobacillus johnsonii and CAG-1031 spp.) 
TABLE 3 | A summary of preclinical and human studies on the gut microbiota and spinal cord injury.

Subjects Methods $\quad$ Key findings

Gungor B, 30 patients vs. 10 controls

et al.

(2016)

(108)

\section{Zhang C, 43 patients vs. 23 controls} et al.

(2018)

(109)

Lin R, et al. 23 patients vs. 23 controls (2020)

(110)

Li J, et al. 32 patients (7 acute SCl and

(2020) 25 long-standing SCl) vs.

(111) 25controls

Bazzocchi 100 patients

$\mathrm{G}$, et al.

(2021)

(112)

Yu B, et al. 45 patients (21 complete (2021) thoracic SCl and 24 incomplete thoracic SCI) vs. 24 controls

Kigerl KA, T9 contusion mice model et al. (2016) (25)

\section{O'Connor T9 contusion rat model}

$\mathrm{G}$, et al.

(2018)

(114)

Myers SA, T9 contusion mice model

et al.

(2019)

(115)

Jing $Y$,

et al.

(2019)

(116)

Schmidt C5 contusion rat model

EKA, et al.

(2020)

(117)
$16 S$ rRNA (V4) - Marvinbryantia was significantly lower in the upper motor neuron (UMN) bowel dysfunction group sequencing than in the lower motor neuron (LMN) group after spinal cord injury(SCl).

- Compared with healthy groups, Roseburia, Pseudobutyrivibrio, and Megamonas were significantly lower in the LMN bowel dysfunction group; the abundances of Pseudobutyrivibrio, Dialister, and Megamonas genera were significantly lower in the UMN bowel dysfunction group.

16S rRNA (V3-V4) - SCl contributed to the increased abundance of Veillonellaceae and Prevotellaceae and the sequencing decreased abundance of Bacteroidaceae and Bacteroides.

- The abundance of Bacteroidaceae and Bacteroides in the quadriplegia group and Acidaminococcaceae, Blautia, Porphyromonadaceae, and Lachnoclostridium in the paraplegia group were significantly higher compared to the healthy male group.

- Following $\mathrm{SCl}$, the gut microbiota composition was significantly associated with serum biomarkers (glucose, high-density lipoprotein, creatinine, and C-reactive protein), neurogenic bowel dysfunction defecation time, and course.

$16 \mathrm{~S}$ rRNA (V3-V4) - There were no significant differences in the $\alpha$-diversity indices of the fecal microbiota between the sequencing $\mathrm{SCl}$ and control groups.

- The abundances of Parabacteroides, Alistipes, Phascolarctobacterium, Christensenella, Barnesiella, Holdemania, Eggerthella, Intestinimonas, Gordonibacter, Bilophila, Flavonifractor, and Coprobacillus were higher in the patients with SCI than those in the healthy control.

16S rRNA (V4) - Compared with the controls, SCl patients had higher abundances of the Erysipelotrichaceae, sequencing Acidaminococcaceae, Rikenellaceae, Lachnospiraceae, Rikenellaceae, the Ruminococcaceae families.

- The long-standing SCl patients had higher abundances of Lachnospiraceae and Eggerthellaceae, and lower abundances of Campylobacteraceae than the controls.

- The acute SCl has a higher abundance of the Desulfovibrionaceae family than the controls. 16S rRNA (V3-V4) - The SCl-induced gut microbiota composition showed distinct dysbiotic signatures with an sequencing enriched in potentially pathogenic, pro-inflammatory, and mucus-degrading bacteria and a decreased abundance of short-chain fatty acids (SCFAs) producers.

- The gut microbiota dysbiosis is very likely secondary to injury and closely related to the lesion's degree of completeness and severity after SCl.

$16 \mathrm{~S}$ rRNA - Compared with healthy individuals, Actinobacteria and Synergistetes were significantly enriched in sequencing patients with complete thoracic SCl. Similarly, Bacteroidetes, Cyanobacteria, and Proteobacteria were significantly lower in patients with incomplete thoracic SCl than healthy controls.

- $\quad$ Coriobacteriaceae, Synergistetes, Eubacterium, and Cloacibacillus, were significantly increased in patients with complete thoracic SCI, while Lactobacillaceae, Lachnospiraceae, Eubacterium, Clostridium, and Sutterella, were significantly increased in patients with incomplete thoracic SCl.

$16 \mathrm{~S}$ rRNA (V4-V5) - SCl increased intestinal permeability and bacterial translocation from the gut, associated with sequencing immune cell activation in gut-associated lymphoid tissues (GALTS) and the altered gut microbiota composition.

- In naive mice, gut dysbiosis induced by oral delivery of broad-spectrum antibiotics before $\mathrm{SCl}$ exacerbated neurological impairment and spinal cord pathology after SCI.

- $\mathrm{SCl}$ mice treated by commercial probiotics (VSL\#3) enriched lactic acid-producing bacteria, triggering a protective immune response in GALTs and conferring neuroprotection with improved neurological recovery.

$16 \mathrm{~S}$ rRNA (V4) - Lactobacillus intestinalis, Clostridium disporicum, and Bifidobacterium choerinum were enriched, sequencing while Clostridium saccharogumia was depleted following SCI.

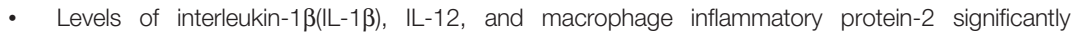
correlated with changes in $\beta$ diversity 8 -weeks post-SCl.

$16 \mathrm{~S}$ rRNA (V4) - $\quad$ SCl led to an increased abundance of Proteobacteria.

sequencing - The absence of Pde4b improved white matter sparing and recovery of hindlimb locomotion following SCl. Moreover, SCl-induced gut dysbiosis, bacterial overgrowth, and endotoxemia were also reduced in $\mathrm{Pde} 4 \mathrm{~b}^{-1-}$ mice.

16S rRNA (V3-V4) • Daily intraperitoneal injection with melatonin improved enhanced barrier integrity and sequencing gastrointestinal motility, reduced proinflammatory cytokines, and promoted locomotor recovery.

- Melatonin-treated $\mathrm{SCl}$ animals decreased the abundance of Clostridiales and increased the quantity of Lactobacillales and Lactobacillus.

- Before surgery, gut dysbiosis induced by broad-spectrum antibiotics exacerbated neurological impairment following SCl, and melatonin treatment improved locomotor recovery and intestinal integrity in antibiotic-treated SCl mice.

$16 \mathrm{~S}$ rRNA (V4) - S SCl-induced dysbiosis increased symptoms of anxiety-like behavior.

sequencing - Fecal microbiota transplantation (FMT) prevented SCl-induced dysbiosis and the development of anxiety-like behavior. 
TABLE 3 | Continued

\begin{tabular}{|c|c|c|c|}
\hline & Subjects & Methods & Key findings \\
\hline $\begin{array}{l}\text { Jing } Y \text {, } \\
\text { et al. } \\
(2021) \\
(118)\end{array}$ & T10 contusion mice model & $\begin{array}{l}\text { 16S rRNA (V4) } \\
\text { sequencing \& } \\
\text { HPLC-MS }\end{array}$ & $\begin{array}{l}\text { - FMT-treated } \mathrm{SCl} \text { mice facilitated functional recovery, promoted neuronal axonal regeneration, and } \\
\text { enhanced intestinal barrier integrity and gastrointestinal motility, which short-chain fatty acids } \\
\text { (SCFAs) and Nuclear Factor- } \mathrm{KB}(\mathrm{NF}-\mathrm{\kappa B}) \text { signaling may mediate. } \\
\text { - } \quad \text { Butyricimonas were reduced in SCI mice, and FMT significantly reshaped gut microbiota. }\end{array}$ \\
\hline $\begin{array}{l}\text { Schmidt } \\
\text { EKA, et al. } \\
(2021) \\
(119)\end{array}$ & C5 contusion rat model & $\begin{array}{l}\text { 16S rRNA (V4) } \\
\text { sequencing }\end{array}$ & $\begin{array}{l}\text { - Minocycline had a profound acute effect on the gut microbiota diversity and composition after } \\
\text { - GCl. } \\
\text { Gut dysbiosis following SCI has been linked to the development of anxiety-like behavior, which } \\
\text { was also alleviated by minocycline. } \\
\text { - Although minocycline attenuated SCl-induced microglial activation, it did not change the lesion } \\
\text { size or promote neurological recovery. }\end{array}$ \\
\hline $\begin{array}{l}\text { Doelman } \\
\text { A, et al. } \\
(2021) \\
(120)\end{array}$ & $\begin{array}{l}\text { T2 or T10 contusion pig } \\
\text { model }\end{array}$ & $\begin{array}{l}16 \mathrm{~S} \text { rRNA (V3-V4) } \\
\text { sequencing }\end{array}$ & $\begin{array}{l}\text { - In the acute phase }(<14 \mathrm{~d} \text { post-SCI), Proteobacteria, Tenericutes, Epsilonbacteraeota, and } \\
\text { Cyanobacteria decreased compared to the controls while Bacteroidetes, Firmicutes, and } \\
\text { Spirochaetes were enriched. } \\
\text { - In the sub-acute phase ( }>14 \text { days post-SCI), the abundance of Spirochaetes, Cyanobacteria, and } \\
\text { Proteobacteria remained statistically significantly different from the controls. }\end{array}$ \\
\hline $\begin{array}{l}\text { Rong Z, } \\
\text { et al. } \\
(2021) \\
(121)\end{array}$ & T10 contusion mice model & - & $\begin{array}{l}\text { - The levels of pro-inflammatory cytokines tumor necrosis factor- } \alpha, \mathrm{IL}-1 \beta \text {, and IL- } 6 \text { in } \mathrm{SCl} \text { mice } \\
\text { were increased, while the levels of anti-inflammatory factors IL-4, transforming growth factor- } \beta \text {, } \\
\text { and IL-10 were decreased. } \\
\text { Gut microbiota dysbiosis aggravated } \mathrm{SCl} \text { by activating the toll-like receptor } 4(T L R 4) / \text { myeloid } \\
\text { differentiation factor } 88 \text { (MyD88) signaling pathway. }\end{array}$ \\
\hline $\begin{array}{l}\text { Du J, et al. } \\
(2021) \\
(122)\end{array}$ & $\begin{array}{l}\text { T4 or T10 contusion mice } \\
\text { model }\end{array}$ & $\begin{array}{l}\text { genome- and } \\
\text { gene-resolved } \\
\text { metagenomic } \\
\text { analysis }\end{array}$ & $\begin{array}{l}\text { - The abundance of Lactobacillus johnsonii and CAG-1031 spp. decreased, while Weissella } \\
\text { cibaria, Lactococcus lactis_A, Bacteroides thetaiotaomicron were enriched after SCI. } \\
\text { - Microbial-mediated biosynthesis of tryptophan, vitamin B6, and folate was reduced after SCl. } \\
1028 \text { mostly novel viral populations were recovered, which expanded known murine gut viral } \\
\text { species sequence space. } \\
\text { - Phages of beneficial commensal hosts, including CAG-1031, Lactobacillus, and Turicibacter, } \\
\text { decreased, while phages of pathogenic hosts, including Weissella, Lactococcus, and class } \\
\text { Clostridia, increased after SCl. }\end{array}$ \\
\hline
\end{tabular}

16 S rRNA, 16 S ribosomal RNA.

HPLC-MS, high-performance liquid chromatography-mass spectrometry.

significantly decreased, while potentially pathogenic bacteria (Weissella cibaria, Lactococcus lactis_A, Bacteroides thetaiotaomicron) increased after SCI. Functionally, tryptophan, vitamin B6, and folate biosynthesis, encoded by microbial genes, were reduced in the feces after SCI. Interestingly, the study performed by $\mathrm{Du}$ et al. reported that phages of beneficial commensal hosts (CAG-1031, Lactobacillus, and Turicibacter) decreased. In contrast, phages of pathogenic hosts (Weissella, Lactococcus, and class Clostridia) increased after SCI (122). In a Yucatan minipig model with a contusioncompression SCI at T2 or T10, Doelman et al. presented a dynamic view of the microbiome changes following SCI and identified acute stage, 0 - 14 post-SCI, as a special time-frame that many of the bacterial fluctuations occur before returning to "baseline" levels (120).

SCI promotes intestinal leakiness and bacterial translocation associated with activation of immune cells in GALTs, by increasing the population of $\mathrm{B}$ cells, $\mathrm{CD}^{+} \mathrm{T}$ cells, DCs, and macrophages and decreasing $\mathrm{CD} 4^{+} \mathrm{T}$ cell counts (25). $\gamma \delta \mathrm{T}$ celldeficient mice improved functional recovery after SCI (124). Moreover, changes in gut microbiota composition following SCI could predict locomotor impairment (125). Additionally, gut microbiota dysbiosis can aggravate SCI by activating the TLR4/ Myeloid differentiation factor 88 signaling pathway (121).

SCI mice fed with commercial probiotics (VSL\#3) reduced neuropathology, improved locomotor recovery, and promoted an anti-inflammatory response by increasing the number of Treg cells in GALTs (25). Additionally, SCI mice daily treated with melatonin improved gut barrier integrity and functional recovery by reducing the abundance of Clostridiales and enhancing the quantity of Lactobacillales and Lactobacillus, which were related to a more favorable cytokine profile (116). Lactic acid supplementation was also proved to improve functional recovery following SCI (25). FMT prevented both SCI-induced dysbiosis, locomotor function, and the development of anxietylike behavior (117). FMT could increase the amount of fecal SCFAs and downregulate IL- $1 \beta / N F-\kappa B$ signaling in the spinal cord and NF- $\kappa B$ signaling in the gut following SCI (118). A recent study also reported that minocycline treatment attenuated SCI-induced anxiety-like behavior and systemic inflammatory response via altering the Firmicutes/Bacteroidetes ratio (119). Engineered liposomes targeting the MGBA may also be a potential treatment (126).

\section{CONCLUSION AND PERSPECTIVE}

Gut microbiota is closely involved in the development and progression of acute CNS disease through multiple mechanisms, including immunological, endocrine, metabolic, and neural pathways. FMT and probiotics significantly improve brain injury by restoring the acute CNS injuryinduced gut microbiota dysbiosis. Gut microbiota may be a potential target to assist in the treatment of acute CNS injury. 
However, several aspects are still needed to ponder despite a growing number of studies concerning the gut microbiota. Firstly, human gut microbiota composition is different from rodents. Although Firmicutes and Bacteroidetes are the most abundant microbiota both in mice and humans, more than $80 \%$ of the bacteria found in the mice intestine are not colonized in the human intestines based on genus level (127). Secondly, immunological features are also different between rodents and humans. A previous study suggests that the intestinal properties of humans are similar to those of mice. However, differences in intestinal immunity between mice and humans have already been found that $\gamma \delta \mathrm{T}$ cells are found significantly less frequently in the intraepithelial compartment of humans than in mice (128). Thirdly, the effects of enteroviruses, fungi, and bacteriophages cannot be ignored. Bacteriophages have high host specificity that shapes the gut microbiota composition and regulates the host immune response by altering bacterial pathogen-associated molecular patterns and maintaining the host mucosal barrier (129). Although the effects of enteroviruses on health and disease are still unclear, phagevirus-fungi-bacterial-host interaction in the gut should also be considered. Moreover, their role in human acute brain injury or animal models has not been studied so far. Fourthly, developmental disturbances in GF mice should be considered.

\section{REFERENCES}

1. Sun P, Liu DZ, Jickling GC, Sharp FR, Yin KJ. Microrna-Based Therapeutics in Central Nervous System Injuries. J Cereb Blood Flow Metab (2018) 38 (7):1125-48. doi: 10.1177/0271678x18773871

2. Wang Y, Tan H, Hui X. Biomaterial Scaffolds in Regenerative Therapy of the Central Nervous System. BioMed Res Int (2018) 2018(2):7848901-19. doi: $10.1155 / 2018 / 7848901$

3. Sorby-Adams AJ, Marcoionni AM, Dempsey ER, Woenig JA, Turner RJ. The Role of Neurogenic Inflammation in Blood-Brain Barrier Disruption and Development of Cerebral Oedema Following Acute Central Nervous System (CNS) Injury. IJMS (2017) 18(8):1788. doi: 10.3390/ijms18081788

4. Zmora N, Suez J, Elinav E. You are What You Eat: Diet, Health and the Gut Microbiota. Nat Rev Gastroenterol Hepatol (2019) 16(1):35-56. doi: 10.1038/s41575-018-0061-2

5. O'Toole PW, Jeffery IB. Gut Microbiota and Aging. Sci (New York NY) (2015) 350(6265):1214-5. doi: 10.1126/science.aac8469

6. Sánchez B, Delgado S, Blanco-Míguez A, Lourenço A, Gueimonde M, Margolles A. Probiotics, Gut Microbiota, and Their Influence on Host Health and Disease. Mol Nutr Food Res (2017) 61(1):1600240. doi: 10.1002/ mnfr. 201600240

7. Järbrink-Sehgal E, Andreasson A. The Gut Microbiota and Mental Health in Adults. Curr Opin Neurobiol (2020) 62:102-14. doi: 10.1016/j.conb.2020.01.016

8. Sender R, Fuchs S, Milo R. Revised Estimates for the Number of Human and Bacteria Cells in the Body. PloS Biol (2016) 14(8):e1002533. doi: 10.1371/ journal.pbio. 1002533

9. Flowers SA, Ellingrod VL. The Microbiome in Mental Health: Potential Contribution of Gut Microbiota in Disease and Pharmacotherapy Management. Pharmacotherapy (2015) 35(10):910-6. doi: 10.1002/ phar. 1640

10. Arumugam M, Raes J, Pelletier E, Le Paslier D, Yamada T, Mende DR, et al. Enterotypes of the Human Gut Microbiome. Nature (2011) 473(7346):17480. doi: $10.1038 /$ nature 09944

11. Tyler Patterson T, Grandhi R. Gut Microbiota and Neurologic Diseases and Injuries. Adv Exp Med Biol (2020) 1238:73-91. doi: 10.1007/978-981-15-2385-4_6
GF mice have hypoplastic immune structures and differ from SPF mice in the intestinal immune cell populations, such as IgAproducing plasma cells and lamina propria $\mathrm{CD} 4^{+} \mathrm{T}$ cells (130). Additionally, GF mice contain fewer serum immunoglobulins, particularly IgG (131). In the absence of gut microbiota, CNS is also altered with a "leaky" BBB and an abnormal microglia morphology and function (132, 133). Finally, criteria for identifying qualified, healthy donors in the FMT treatment have not yet been fully established. The safety and efficiency of FMT need to be extensively investigated.

\section{AUTHOR CONTRIBUTIONS}

BY wrote the manuscript. X-jL and QW revised the manuscript. All authors contributed to the article and approved the submitted version.

\section{FUNDING}

This work was supported by the Scientific Research Project of Jiangsu Provincial Health commission under Grant No. H2018066.

12. Takagi T, Naito Y, Inoue R, Kashiwagi S, Uchiyama K, Mizushima K, et al. Differences in Gut Microbiota Associated With Age, Sex, and Stool Consistency in Healthy Japanese Subjects. J Gastroenterol (2019) 54(1):5363. doi: 10.1007/s00535-018-1488-5

13. Delgado Jiménez R, Benakis C. The Gut Ecosystem: A Critical Player in Stroke. Neuromolecular Med. (2021) 23(2):236-241. doi: 10.1007/s12017020-08633-z

14. Knight R, Vrbanac A, Taylor BC, Aksenov A, Callewaert C, Debelius J, et al. Best Practices for Analysing Microbiomes. Nat Rev Microbiol (2018) 16 (7):410-22. doi: 10.1038/s41579-018-0029-9

15. Arya AK, Hu B. Brain-Gut Axis After Stroke. Brain Circ (2018) 4(4):165-73. doi: 10.4103/bc.bc_32_18

16. Russo R, Cristiano C, Avagliano C, De Caro C, La Rana G, Raso GM, et al. Gut-Brain Axis: Role of Lipids in the Regulation of Inflammation, Pain and CNS Diseases. Curr Med Chem (2018) 25(32):3930-52. doi: 10.2174/ 0929867324666170216113756

17. Fung TC, Olson CA, Hsiao EY. Interactions Between the Microbiota, Immune and Nervous Systems in Health and Disease. Nat Neurosci (2017) 20(2):145-55. doi: 10.1038/nn.4476

18. Cryan JF, O'Riordan KJ, Cowan CSM, Sandhu KV, Bastiaanssen TFS, Boehme M, et al. The Microbiota-Gut-Brain Axis. Physiol Rev (2019) 99 (4):1877-2013. doi: 10.1152/physrev.00018.2018

19. Benakis C, Martin-Gallausiaux C, Trezzi JP, Melton P, Liesz A, Wilmes P. The Microbiome-Gut-Brain Axis in Acute and Chronic Brain Diseases. Curr Opin Neurobiol (2020) 61:1-9. doi: 10.1016/j.conb.2019.11.009

20. Cryan JF, O'Riordan KJ, Sandhu K, Peterson V, Dinan TG. The Gut Microbiome in Neurological Disorders. Lancet Neurol (2020) 19(2):17994. doi: 10.1016/S1474-4422(19)30356-4

21. Houlden A, Goldrick M, Brough D, Vizi ES, Lénárt N, Martinecz B, et al. Brain Injury Induces Specific Changes in the Caecal Microbiota of Mice via Altered Autonomic Activity and Mucoprotein Production. Brain Behav Immun (2016) 57:10-20. doi: 10.1016/j.bbi.2016.04.003

22. Antila S, Karaman S, Nurmi H, Airavaara M, Voutilainen MH, Mathivet T, et al. Development and Plasticity of Meningeal Lymphatic Vessels. J Exp Med (2017) 214(12):3645-67. doi: 10.1084/jem.20170391 
23. Singh V, Sadler R, Heindl S, Llovera G, Roth S, Benakis C, et al. The Gut Microbiome Primes a Cerebroprotective Immune Response After Stroke. J Cereb Blood Flow Metab (2018) 38(8):1293-8. doi: 10.1177/ 0271678X18780130

24. Brea D, Poon C, Benakis C, Lubitz G, Murphy M, Iadecola C, et al. Stroke Affects Intestinal Immune Cell Trafficking to the Central Nervous System. Brain Behav Immun (2021) 96:295-302. doi: 10.1016/j.bbi.2021.05.008

25. Kigerl KA, Hall JCE, Wang L, Mo X, Yu Z, Popovich PG. Gut Dysbiosis Impairs Recovery After Spinal Cord Injury. J Exp Med (2016) 213(12):260320. doi: $10.1084 /$ jem.20151345

26. Wen L, You W, Wang H, Meng Y, Feng J, Yang X. Polarization of Microglia to the M2 Phenotype in a Peroxisome Proliferator-Activated Receptor Gamma-Dependent Manner Attenuates Axonal Injury Induced by Traumatic Brain Injury in Mice. J neurotrauma (2018) 35(19):2330-40. doi: 10.1089/neu.2017.5540

27. Carlson AL, Xia K, Azcarate-Peril MA, Goldman BD, Ahn M, Styner MA, et al. Infant Gut Microbiome Associated With Cognitive Development. Biol Psychiatry (2018) 83(2):148-59. doi: 10.1016/j.biopsych.2017.06.021

28. Gao W, Salzwedel AP, Carlson AL, Xia K, Azcarate-Peril MA, Styner MA, et al. Gut Microbiome and Brain Functional Connectivity in Infants-A Preliminary Study Focusing on the Amygdala. Psychopharmacology (2019) 236(5):1641-51. doi: 10.1007/s00213-018-5161-8

29. Harms AS, Thome AD, Yan Z, Schonhoff AM, Williams GP, Li X, et al. Peripheral Monocyte Entry Is Required for Alpha-Synuclein Induced Inflammation and Neurodegeneration in a Model of Parkinson Disease. Exp Neurol (2018) 300:179-87. doi: 10.1016/j.expneurol.2017.11.010

30. Mazmanian SK, Liu CH, Tzianabos AO, Kasper DL. An Immunomodulatory Molecule of Symbiotic Bacteria Directs Maturation of the Host Immune System. Cell (2005) 122(1):107-18. doi: 10.1016/ j.cell.2005.05.007

31. Atarashi K, Suda W, Luo C, Kawaguchi T, Motoo I, Narushima S, et al. Ectopic Colonization of Oral Bacteria in the Intestine Drives TH1 Cell Induction and Inflammation. Science (2017) 358(6361):359-65. doi: $10.1126 /$ science.aan4526

32. Fort MM, Cheung J, Yen D, Li J, Zurawski SM, Lo S, et al. IL-25 Induces IL4, IL-5, and IL-13 and Th2-Associated Pathologies In Vivo. Immunity (2001) 15(6):985-95. doi: 10.1016/s1074-7613(01)00243-6

33. Wang Y, Yin Y, Chen X, Zhao Y, Wu Y, Li Y, et al. Induction of Intestinal Th17 Cells by Flagellins From Segmented Filamentous Bacteria. Front Immunol (2019) 10:2750. doi: 10.3389/fimmu.2019.02750

34. Round JL, Lee SM, Li J, Tran G, Jabri B, Chatila TA, et al. The Toll-Like Receptor 2 Pathway Establishes Colonization by a Commensal of the Human Microbiota. Sci (New York NY) (2011) 332(6032):974-7. doi: 10.1126/science.1206095

35. Fleming C, Cai Y, Sun X, Jala VR, Xue F, Morrissey S, et al. MicrobiotaActivated CD103(+) Dcs Stemming From Microbiota Adaptation Specifically Drive Gammadeltat 17 Proliferation and Activation. Microbiome (2017) 5(1):46. doi: 10.1186/s40168-017-0263-9

36. Yang $\mathrm{Y}, \mathrm{Xu} \mathrm{C}, \mathrm{Wu} \mathrm{D}$, Wang Z, Wu P, Li L, et al. $\Gamma \delta$ T Cells: Crosstalk Between Microbiota, Chronic Inflammation, and Colorectal Cancer. Front Immunol (2018) 9:1483. doi: 10.3389/fimmu.2018.01483

37. Wesemann DR, Portuguese AJ, Meyers RM, Gallagher MP, Cluff-Jones K, Magee JM, et al. Microbial Colonization Influences Early B-Lineage Development in the Gut Lamina Propria. Nature (2013) 501(7465):112-5. doi: $10.1038 /$ nature 12496

38. Shaw MH, Kamada N, Kim Y-G, Núñez G. Microbiota-Induced IL-1ß, But Not IL-6, Is Critical for the Development of Steady-State TH17 Cells in the Intestine. J Exp Med (2012) 209(2):251-8. doi: 10.1084/jem.20111703

39. Chang PV, Hao L, Offermanns S, Medzhitov R. The Microbial Metabolite Butyrate Regulates Intestinal Macrophage Function via Histone Deacetylase Inhibition. Proc Natl Acad Sci United States America (2014) 111(6):2247-52. doi: $10.1073 /$ pnas.1322269111

40. Singh N, Gurav A, Sivaprakasam S, Brady E, Padia R, Shi H, et al. Activation of Gpr109a, Receptor for Niacin and the Commensal Metabolite Butyrate, Suppresses Colonic Inflammation and Carcinogenesis. Immunity (2014) 40 (1):128-39. doi: 10.1016/j.immuni.2013.12.007

41. Parada Venegas D, de la Fuente MK, Landskron G, Gonzalez MJ, Quera R, Dijkstra G, et al. Short Chain Fatty Acids (Scfas)-Mediated Gut Epithelial and Immune Regulation and Its Relevance for Inflammatory Bowel Diseases. Front Immunol (2019) 10:277. doi: 10.3389/fimmu.2019.00277

42. Sun X, Jiao X, Ma Y, Liu Y, Zhang L, He Y, et al. Trimethylamine N-Oxide Induces Inflammation and Endothelial Dysfunction in Human Umbilical Vein Endothelial Cells via Activating ROS-TXNIP-NLRP3 Inflammasome. Biochem Biophys Res Commun (2016) 481(1-2):63-70. doi: 10.1016/ j.bbrc.2016.11.017

43. Dasgupta S, Erturk-Hasdemir D, Ochoa-Reparaz J, Reinecker H-C, Kasper DL. Plasmacytoid Dendritic Cells Mediate Anti-Inflammatory Responses to a Gut Commensal Molecule via Both Innate and Adaptive Mechanisms. Cell Host Microbe (2014) 15(4):413-23. doi: 10.1016/j.chom.2014.03.006

44. Johnson JL, Jones MB, Cobb BA. Polysaccharide a From the Capsule of Bacteroides Fragilis Induces Clonal CD4+ T Cell Expansion. J Biol Chem (2015) 290(8):5007-14. doi: 10.1074/jbc.M114.621771

45. Wang Y, Begum-Haque S, Telesford KM, Ochoa-Reparaz J, Christy M, Kasper EJ, et al. A Commensal Bacterial Product Elicits and Modulates Migratory Capacity of CD39(+) CD4 T Regulatory Subsets in the Suppression of Neuroinflammation. Gut Microbes (2014) 5(4):552-61. doi: $10.4161 /$ gmic. 29797

46. Feigin VL, Norrving B, Mensah GA. Global Burden of Stroke. Circ Res (2017) 120(3):439-48. doi: 10.1161/CIRCRESAHA.116.308413

47. Ojaghihaghighi S, Vahdati SS, Mikaeilpour A, Ramouz A. Comparison of Neurological Clinical Manifestation in Patients With Hemorrhagic and Ischemic Stroke. World J Emerg Med (2017) 8(1):34-8. doi: 10.5847/ wjem.j.1920-8642.2017.01.006

48. Yin J, Liao S-X, He Y, Wang S, Xia G-H, Liu F-T, et al. Dysbiosis of Gut Microbiota With Reduced Trimethylamine-N-Oxide Level in Patients With Large-Artery Atherosclerotic Stroke or Transient Ischemic Attack. J Am Heart Assoc (2015) 4(11):e002699. doi: 10.1161/JAHA.115.002699

49. Haak BW, Westendorp WF, van Engelen TSR, Brands X, Brouwer MC, Vermeij J-D, et al. Disruptions of Anaerobic Gut Bacteria Are Associated With Stroke and Post-Stroke Infection: A Prospective Case-Control Study. Trans Stroke Res (2020) 11:110-12. doi: 10.1007/s12975-020-00863-4

50. Zeng X, Gao X, Peng Y, Wu Q, Zhu J, Tan C, et al. Higher Risk of Stroke is Correlated With Increased Opportunistic Pathogen Load and Reduced Levels of Butyrate-Producing Bacteria in the Gut. Front Cell Infect Microbiol (2019) 9:4. doi: 10.3389/fcimb.2019.00004

51. Nie J, Xie L, Zhao B-X, Li Y, Qiu B, Zhu F, et al. Serum Trimethylamine NOxide Concentration Is Positively Associated With First Stroke in Hypertensive Patients. Stroke (2018) 49(9):2021-8. doi: 10.1161/ STROKEAHA.118.021997

52. Stanley D, Mason LJ, Mackin KE, Srikhanta YN, Lyras D, Prakash MD, et al. Translocation and Dissemination of Commensal Bacteria in Post-Stroke Infection. Nat Med (2016) 22(11):1277-84. doi: 10.1038/nm.4194

53. Spychala MS, Venna VR, Jandzinski M, Doran SJ, Durgan DJ, Ganesh BP, et al. Age-Related Changes in the Gut Microbiota Influence Systemic Inflammation and Stroke Outcome. Ann Neurol (2018) 84(1):23-36. doi: 10.1002/ana.25250

54. Singh V, Roth S, Llovera G, Sadler R, Garzetti D, Stecher B, et al. Microbiota Dysbiosis Controls the Neuroinflammatory Response After Stroke. J Neurosci (2016) 36(28):7428-40. doi: 10.1523/JNEUROSCI.1114-16.2016

55. Benakis C, Brea D, Caballero S, Faraco G, Moore J, Murphy M, et al. Commensal Microbiota Affects Ischemic Stroke Outcome by Regulating Intestinal $\Gamma \delta$ T Cells. Nat Med (2016) 22(5):516-23. doi: 10.1038/nm.4068

56. Benakis C, Poon C, Lane D, Brea D, Sita G, Moore J, et al. Distinct Commensal Bacterial Signature in the Gut Is Associated With Acute and Long-Term Protection From Ischemic Stroke. Stroke (2020) 51(6):1844-54. doi: 10.1161/STROKEAHA.120.029262

57. Sadler R, Cramer JV, Heindl S, Kostidis S, Betz D, Zuurbier KR, et al. ShortChain Fatty Acids Improve Poststroke Recovery via Immunological Mechanisms. J Neurosci (2020) 40(5):1162-73. doi: 10.1523/ JNEUROSCI.1359-19.2019

58. Lee J, d'Aigle J, Atadja L, Quaicoe V, Honarpisheh P, Ganesh BP, et al. Gut Microbiota-Derived Short-Chain Fatty Acids Promote Poststroke Recovery in Aged Mice. Circ Res (2020) 127(4):453-65. doi: 10.1161/ CIRCRESAHA.119.316448

59. Winek K, Engel O, Koduah P, Heimesaat MM, Fischer A, Bereswill S, et al. Depletion of Cultivatable Gut Microbiota by Broad-Spectrum Antibiotic 
Pretreatment Worsens Outcome After Murine Stroke. Stroke (2016) 47 (5):1354-63. doi: 10.1161/STROKEAHA.115.011800

60. Xu DJ, Wang KC, Yuan LB, Li HF, Xu YY, Wei LY, et al. Compositional and Functional Alterations of Gut Microbiota in Patients With Stroke. Nutr Metab Cardiovasc Dis (2021) 31(12):3434-48. doi: 10.1016/ j.numecd.2021.08.045

61. Ling Y, Gong T, Zhang J, Gu Q, Gao X, Weng X, et al. Gut Microbiome Signatures Are Biomarkers for Cognitive Impairment in Patients With Ischemic Stroke. Front Aging Neurosci 12:511562. doi: 10.3389/ fnagi.2020.511562

62. Xiang L, Lou Y, Liu L, Liu Y, Zhang W, Deng J, et al. Gut Microbiotic Features Aiding the Diagnosis of Acute Ischemic Stroke. Front Cell Infect Microbiol (2020) 10:587284. doi: 10.3389/fcimb.2020.587284

63. Huang Y, Shen Z, He W. Identification of Gut Microbiome Signatures in Patients With Post-Stroke Cognitive Impairment and Affective Disorder. Front Aging Neurosci (2021) 13:706765. doi: 10.3389/fnagi.2021.706765

64. Xu K, Gao X, Xia G, Chen M, Zeng N, Wang S, et al. Rapid Gut Dysbiosis Induced by Stroke Exacerbates Brain Infarction in Turn. Gut (2021) 70:1486-94. doi: 10.1136/gutjnl-2020-323263

65. Jeon J, Lourenco J, Kaiser EE, Waters ES, Scheulin KM, Fang X, et al. Dynamic Changes in the Gut Microbiome at the Acute Stage of Ischemic Stroke in a Pig Model. Front Neurosci (2020) 14:587986. doi: 10.3389/ fnins. 2020.587986

66. Wu W, Sun Y, Luo N, Cheng C, Jiang C, Yu Q, et al. Integrated 16S Rrna Gene Sequencing and LC-MS Analysis Revealed the Interplay Between Gut Microbiota and Plasma Metabolites in Rats With Ischemic Stroke. J Mol Neurosci (2021) 71(10):2095-106. doi: 10.1007/s12031-021-01828-4

67. Tan C, Wu Q, Wang H, Gao X, Xu R, Cui Z, et al. Dysbiosis of Gut Microbiota and Short-Chain Fatty Acids in Acute Ischemic Stroke and the Subsequent Risk for Poor Functional Outcomes. JPEN J Parenter Enteral Nutr (2021) 45(3):518-29. doi: 10.1002/jpen.1861

68. Zhang J, Wang L, Cai J, Lei A, Liu C, Lin R, et al. Gut Microbial Metabolite TMAO Portends Prognosis in Acute Ischemic Stroke. J Neuroimmunol (2021) 354:577526. doi: 10.1016/j.jneuroim.2021.577526

69. Guo Q, Jiang X, Ni C, Li L, Chen L, Wang Y, et al. Gut Microbiota-Related Effects of Tanhuo Decoction in Acute Ischemic Stroke. Oxid Med Cell Longev (2021) 2021:5596924. doi: 10.1155/2021/5596924

70. Sun T, Zhang Y, Yin J, Peng X, Zhou L, Huang S, et al. Association of Gut Microbiota-Dependent Metabolite Trimethylamine N-Oxide With First Ischemic Stroke. J Atheroscler Thromb (2021) 28(4):320-8. doi: 10.5551/ jat.55962

71. Huang Q, Di L, Yu F, Feng X, Liu Z, Wei M, et al. Alterations in the Gut Microbiome With Hemorrhagic Transformation in Experimental Stroke. CNS Neurosci Ther (2022) 28(1):77-91. doi: 10.1111/cns.13736

72. Zhang P, Zhang X, Huang Y, Chen J, Shang W, Shi G, et al. Atorvastatin Alleviates Microglia-Mediated Neuroinflammation via Modulating the Microbial Composition and the Intestinal Barrier Function in Ischemic Stroke Mice. Free Radic Biol Med (2021) 162:104-17. doi: 10.1016/ j.freeradbiomed.2020.11.032

73. Huang JT, Mao YQ, Han B, Zhang ZY, Chen HL, Li ZM, et al. Calorie Restriction Conferred Improvement Effect on Long-Term Rehabilitation of Ischemic Stroke via Gut Microbiota. Pharmacol Res (2021) 170:105726. doi: 10.1016/j.phrs.2021.105726

74. Zhu W, Romano KA, Li L, Buffa JA, Sangwan N, Prakash P, et al. Gut Microbes Impact Stroke Severity via the Trimethylamine N-Oxide Pathway. Cell Host Microbe (2021) 29(7):1199-208. doi: 10.1016/j.chom.2021.05.002

75. Yuan Q, Xin L, Han S, Su Y, Wu R, Liu X, et al. Lactulose Improves Neurological Outcomes by Repressing Harmful Bacteria and Regulating Inflammatory Reactions in Mice After Stroke. Front Cell Infect Microbiol (2021) 11:644448. doi: 10.3389/fcimb.2021.644448

76. Li N, Wang X, Sun C, Wu X, Lu M, Si Y, et al. Change of Intestinal Microbiota in Cerebral Ischemic Stroke Patients. BMC Microbiol (2019) 19 (1):191-8. doi: 10.1186/s12866-019-1552-1

77. Sun J, Ling Z, Wang F, Chen W, Li H, Jin J, et al. Clostridium Butyricum Pretreatment Attenuates Cerebral Ischemia/Reperfusion Injury in Mice via Anti-Oxidation and Anti-Apoptosis. Neurosci Lett (2016) 613:30-5. doi: 10.1016/j.neulet.2015.12.047
78. Zhou Z, Xu N, Matei N, McBride DW, Ding Y, Liang H, et al. Sodium Butyrate Attenuated Neuronal Apoptosis via GPR41/Gbetagamma/PI3K/ Akt Pathway After MCAO in Rats. J Cereb Blood Flow Metab (2021) 41 (2):267-81. doi: 10.1177/0271678X20910533

79. Yan H, Ajuwon KM. Butyrate Modifies Intestinal Barrier Function in IPECJ2 Cells Through a Selective Upregulation of Tight Junction Proteins and Activation of the Akt Signaling Pathway. PloS One (2017) 12(6):e0179586. doi: 10.1371/journal.pone. 0179586

80. Wu C, Li C, Zhao W, Xie N, Yan F, Lian Y, et al. Elevated Trimethylamine N-Oxide Related to Ischemic Brain Lesions After Carotid Artery Stenting. Neurology (2018) 90(15):e1283-90. doi: 10.1212/WNL.0000000000005298

81. Tan C, Wang H, Gao X, Xu R, Zeng X, Cui Z, et al. Dynamic Changes and Prognostic Value of Gut Microbiota-Dependent Trimethylamine-N-Oxide in Acute Ischemic Stroke. Front Neurol (2020) 11:2020.00029. doi: 10.3389/ fneur.2020.00029

82. Farhangi MA, Vajdi M, Asghari-Jafarabadi M. Gut Microbiota-Associated Metabolite Trimethylamine N-Oxide and the Risk of Stroke: A Systematic Review and Dose-Response Meta-Analysis. Nutr J (2020) 19(1):76. doi: 10.1186/s12937-020-00592-2

83. Chen W-C, Chang L-H, Huang S-S, Huang Y-J, Chih C-L, Kuo H-C, et al. Aryl Hydrocarbon Receptor Modulates Stroke-Induced Astrogliosis and Neurogenesis in the Adult Mouse Brain. J Neuroinflamm (2019) 16 (1):187-13. doi: 10.1186/s12974-019-1572-7

84. Macdonald RL, Schweizer TA. Spontaneous Subarachnoid Haemorrhage. Lancet (2017) 389(10069):655-66. doi: 10.1016/S0140-6736(16)30668-7

85. Li H, Xu H, Li Y, Jiang Y, Hu Y, Liu T, et al. Alterations of Gut Microbiota Contribute to the Progression of Unruptured Intracranial Aneurysms. Nat Commun (2020) 11(1):3218. doi: 10.1038/s41467-020-16990-3

86. Kawabata S, Takagaki M, Nakamura H, Oki H, Motooka D, Nakamura S, et al. Dysbiosis of Gut Microbiome is Associated With Rupture of Cerebral Aneurysms. Stroke (2021), STROKEAHA121034792. doi: 10.1161/ STROKEAHA.121.034792

87. Shikata F, Shimada K, Sato H, Ikedo T, Kuwabara A, Furukawa H, et al. Potential Influences of Gut Microbiota on the Formation of Intracranial Aneurysm. Hypertension (2019) 73(2):491-6. doi: 10.1161/ HYPERTENSIONAHA.118.11804

88. Maas AIR, Menon DK, Adelson PD, Andelic N, Bell MJ, Belli A, et al. Traumatic Brain Injury: Integrated Approaches to Improve Prevention, Clinical Care, and Research. Lancet Neurol (2017) 16(12):987-1048. doi: 10.1016/S1474-4422(17)30371-X

89. Simon DW, Rogers MB, Gao Y, Vincent G, Firek BA, Janesko-Feldman K, et al. Depletion of Gut Microbiota Is Associated With Improved Neurologic Outcome Following Traumatic Brain Injury. Brain Res (2020) 1747:147056. doi: 10.1016/j.brainres.2020.147056

90. Mahajan C, Khurana S, Kapoor I, Sokhal S, Kumar S, Prabhakar H, et al. Characteristics of Gut Microbiome After Traumatic Brain Injury. J Neurosurg Anesthesiol (2021). doi: 10.1097/ANA.0000000000000789

91. Hou Y, Xu L, Song S, Fan W, Wu Q, Tong X, et al. Oral Administration of Brain Protein Combined With Probiotics Induces Immune Tolerance Through the Tryptophan Pathway. Front Mol Neurosci (2021) 14:634631. doi: $10.3389 /$ fnmol.2021.634631

92. Treangen TJ, Wagner J, Burns MP, Villapol S. Traumatic Brain Injury in Mice Induces Acute Bacterial Dysbiosis Within the Fecal Microbiome. Front Immunol (2018) 9:2757. doi: 10.3389/fimmu.2018.02757

93. Li H, Sun J, Du J, Wang F, Fang R, Yu C, et al. Clostridium Butyricum Exerts a Neuroprotective Effect in a Mouse Model of Traumatic Brain Injury via the Gut-Brain Axis. Neurogastroenterol Motil (2018) 30(5):e13260. doi: 10.1111/ nmo. 13260

94. Angoa-Pérez M, Zagorac B, Anneken JH, Briggs DI, Winters AD, Greenberg JM, et al. Repetitive, Mild Traumatic Brain Injury Results in a Progressive White Matter Pathology, Cognitive Deterioration, and a Transient Gut Microbiota Dysbiosis. Sci Rep (2020) 10(1):8949. doi: 10.1038/s41598-02065972-4

95. Opeyemi OM, Rogers MB, Firek BA, Janesko-Feldman K, Vagni V, Mullett SJ, et al. Sustained Dysbiosis and Decreased Fecal Short-Chain Fatty Acids After Traumatic Brain Injury and Impact on Neurologic Outcome. J Neurotrauma (2021) 38(18):2610-21. doi: 10.1089/neu.2020.7506 
96. Du D, Tang W, Zhou C, Sun X, Wei Z, Zhong J, et al. Fecal Microbiota Transplantation Is a Promising Method to Restore Gut Microbiota Dysbiosis and Relieve Neurological Deficits After Traumatic Brain Injury. Oxid Med Cell Longevity (2021) 2021:5816837. doi: 10.1155/2021/5816837

97. You W, Zhu Y, Wei A, Du J, Wang Y, Zheng P, et al. Traumatic Brain Injury Induces Gastrointestinal Dysfunction and Dysbiosis of Gut Microbiota Accompanied by Alterations of Bile Acid Profile. J Neurotrauma (2021). doi: 10.1089/neu.2020.7526

98. Celorrio M, Abellanas MA, Rhodes J, Goodwin V, Moritz J, Vadivelu S, et al. Gut Microbial Dysbiosis After Traumatic Brain Injury Modulates the Immune Response and Impairs Neurogenesis. Acta Neuropathol Commun (2021) 9(1):40. doi: 10.1186/s40478-021-01137-2

99. Nicholson SE, Watts LT, Burmeister DM, Merrill D, Scroggins S, Zou Y, et al. Moderate Traumatic Brain Injury Alters the Gastrointestinal Microbiome in a Time-Dependent Manner. Shock (2019) 52(2):240-8. doi: 10.1097/SHK.0000000000001211

100. Blanke EN, Holmes GM, Besecker EM. Altered Physiology of Gastrointestinal Vagal Afferents Following Neurotrauma. Neural Regener Res (2021) 16(2):254-63. doi: 10.4103/1673-5374.290883

101. Hang C-H, Shi J-X, Li J-S, Li W-Q, Yin H-X. Up-Regulation of Intestinal Nuclear Factor Kappa B and Intercellular Adhesion Molecule-1 Following Traumatic Brain Injury in Rats. World J Gastroenterol (2005) 11(8):1149-54. doi: 10.3748/wjg.v11.i8.1149

102. Lu J, Frerich JM, Turtzo LC, Li S, Chiang J, Yang C, et al. Histone Deacetylase Inhibitors Are Neuroprotective and Preserve NGF-Mediated Cell Survival Following Traumatic Brain Injury. Proc Natl Acad Sci United States America (2013) 110(26):10747-52. doi: 10.1073/pnas.1308950110

103. Zhang X, Jiang X. Effects of Enteral Nutrition on the Barrier Function of the Intestinal Mucosa and Dopamine Receptor Expression in Rats With Traumatic Brain Injury. JPEN J Parenter Enteral Nutr (2015) 39(1):11423. doi: $10.1177 / 0148607113501881$

104. Wan G, Wang L, Zhang G, Zhang J, Lu Y, Li J, et al. Effects of Probiotics Combined With Early Enteral Nutrition on Endothelin-1 and C-Reactive Protein Levels and Prognosis in Patients With Severe Traumatic Brain Injury. J Int Med Res (2020) 48(3):300060519888112. doi: 10.1177/ 0300060519888112

105. Tan M, Zhu J-C, Du J, Zhang L-M, Yin H-H. Effects of Probiotics on Serum Levels of Th1/Th2 Cytokine and Clinical Outcomes in Severe Traumatic Brain-Injured Patients: A Prospective Randomized Pilot Study. Crit Care (London England) (2011) 15(6):R290-10. doi: 10.1186/cc10579

106. Bansal V, Costantini T, Ryu SY, Peterson C, Loomis W, Putnam J, et al. Stimulating the Central Nervous System to Prevent Intestinal Dysfunction After Traumatic Brain Injury. J Trauma (2010) 68(5):1059-64. doi: 10.1097/ TA.0b013e3181d 87373

107. Stothers L, Macnab AJ, Mukisa R, Mutabazi S, Bajunirwe F. Traumatic Spinal Cord Injury in Uganda: A Prevention Strategy and Mechanism to Improve Home Care. Int J Epidemiol (2017) 46(4):1086-90. doi: 10.1093/ije/dyx058

108. Gungor B, Adiguzel E, Gursel I, Yilmaz B, Gursel M. Intestinal Microbiota in Patients With Spinal Cord Injury. PloS One (2016) 11(1):e0145878. doi: 10.1371/journal.pone.0145878

109. Zhang C, Zhang W, Zhang J, Jing Y, Yang M, Du L, et al. Gut Microbiota Dysbiosis in Male Patients With Chronic Traumatic Complete Spinal Cord Injury. J Trans Med (2018) 16(1):353-16. doi: 10.1186/s12967-018-1735-9

110. Lin R, Xu J, Ma Q, Chen M, Wang L, Wen S, et al. Alterations in the Fecal Microbiota of Patients With Spinal Cord Injury. PloS One (2020) 15(8): e0236470. doi: 10.1371/journal.pone.0236470

111. Li J, van der Pol W, Eraslan M, McLain A, Cetin H, Cetin B, et al. Comparison of the Gut Microbiome Composition Among Individuals With Acute or Long-Standing Spinal Cord Injury vs. Able-Bodied Controls. J Spinal Cord Med (2020) 4:1-9. doi: 10.1080/ 10790268.2020.1769949

112. Bazzocchi G, Turroni S, Bulzamini MC, D’Amico F, Bava A, Castiglioni M, et al. Changes in Gut Microbiota in the Acute Phase After Spinal Cord Injury Correlate With Severity of the Lesion. Sci Rep (2021) 11(1):12743. doi: 10.1038/s41598-021-92027-z

113. Yu B, Qiu H, Cheng S, Ye F, Li J, Chen S, et al. Profile of Gut Microbiota in Patients With Traumatic Thoracic Spinal Cord Injury and Its Clinical
Implications: A Case-Control Study in a Rehabilitation Setting. Bioengineered (2021) 12(1):4489-99. doi: 10.1080/21655979.2021.1955543

114. O'Connor G, Jeffrey E, Madorma D, Marcillo A, Abreu MT, Deo SK, et al. Investigation of Microbiota Alterations and Intestinal Inflammation PostSpinal Cord Injury in Rat Model. J Neurotrauma (2018) 35(18):2159-66. doi: 10.1089/neu.2017.5349

115. Myers SA, Gobejishvili L, Saraswat Ohri S, Garrett Wilson C, Andres KR, Riegler AS, et al. Following Spinal Cord Injury, PDE4B Drives an Acute, Local Inflammatory Response and a Chronic, Systemic Response Exacerbated by Gut Dysbiosis and Endotoxemia. Neurobiol Dis (2019) 124:353-63. doi: 10.1016/j.nbd.2018.12.008

116. Jing Y, Yang D, Bai F, Zhang C, Qin C, Li D, et al. Melatonin Treatment Alleviates Spinal Cord Injury-Induced Gut Dysbiosis in Mice. J Neurotrauma (2019) 36(18):2646-64. doi: 10.1089/neu.2018.6012

117. Schmidt EKA, Torres-Espin A, Raposo PJF, Madsen KL, Kigerl KA, Popovich PG, et al. Fecal Transplant Prevents Gut Dysbiosis and AnxietyLike Behaviour After Spinal Cord Injury in. PloS One (2020) 15(1):e0226128. doi: 10.1371/journal.pone.0226128

118. Jing Y, Yu Y, Bai F, Wang L, Yang D, Zhang C, et al. Effect of Fecal Microbiota Transplantation on Neurological Restoration in a Spinal Cord Injury Mouse Model: Involvement of Brain-Gut Axis. Microbiome (2021) 9 (1):59-21. doi: 10.1186/s40168-021-01007-y

119. Schmidt EKA, Raposo PJF, Torres-Espin A, Fenrich KK, Fouad K. Beyond the Lesion Site: Minocycline Augments Inflammation and Anxiety-Like Behavior Following SCI in Rats Through Action on the Gut Microbiota. J Neuroinflammation (2021) 18(1):144. doi: 10.1186/s12974-021-02123-0

120. Doelman A, Tigchelaar S, McConeghy B, Sinha S, Keung MS, Manouchehri $\mathrm{N}$, et al. Characterization of the Gut Microbiome in a Porcine Model of Thoracic Spinal Cord Injury. BMC Genomics (2021) 22(1):775. doi: 10.1186/ s12864-021-07979-3

121. Rong Z, Huang Y, Cai H, Chen M, Wang H, Liu G, et al. Gut Microbiota Disorders Promote Inflammation and Aggravate Spinal Cord Injury Through the TLR4/Myd88 Signaling Pathway. Front Nutr (2021) 8:702659. doi: 10.3389/fnut.2021.702659

122. Du J, Zayed AA, Kigerl KA, Zane K, Sullivan MB, Popovich PG. Spinal Cord Injury Changes the Structure and Functional Potential of Gut Bacterial and Viral Communities. mSystems (2021) 6(3):e01356-20. doi: 10.1128/ mSystems.01356-20

123. Kigerl KA, Zane K, Adams K, Sullivan MB, Popovich PG. The Spinal CordGut-Immune Axis as a Master Regulator of Health and Neurological Function After Spinal Cord Injury. Exp Neurol (2020) 323:113085. doi: 10.1016/j.expneurol.2019.113085

124. Sun G, Yang S, Cao G, Wang Q, Hao J, Wen Q, et al. $\Gamma \delta$ T Cells Provide the Early Source of IFN- $\Gamma$ to Aggravate Lesions in Spinal Cord Injury. J Exp Med (2018) 215(2):521-35. doi: 10.1084/jem.20170686

125. Kigerl KA, Mostacada K, Popovich PG. Gut Microbiota are DiseaseModifying Factors After Traumatic Spinal Cord Injury. Neurotherapeutics (2018) 15(1):60-7. doi: 10.1007/s13311-017-0583-2

126. Wang X, Wu J, Liu X, Tang K, Cheng L, Li J, et al. Engineered Liposomes Targeting the Gut-CNS Axis for Comprehensive Therapy of Spinal Cord Injury. J Control Release (2021) 331:390-403. doi: 10.1016/j.jconrel.2021.01.032

127. Ley RE, Bäckhed F, Turnbaugh P, Lozupone CA, Knight RD, Gordon JI. Obesity Alters Gut Microbial Ecology. Proc Natl Acad Sci USA (2005) 102 (31):11070-5. doi: 10.1073/pnas.0504978102

128. Mann ER, Landy JD, Bernardo D, Peake STC, Hart AL, Al-Hassi HO, et al. Intestinal Dendritic Cells: Their Role in Intestinal Inflammation, Manipulation by the Gut Microbiota and Differences Between Mice and Men. Immunol Lett (2013) 150(1-2):30-40. doi: 10.1016/j.imlet.2013.01.007

129. De Paepe M, Leclerc M, Tinsley CR, Petit M-A. Bacteriophages: An Underestimated Role in Human and Animal Health? Front Cell Infect Microbiol (2014) 4:39. doi: 10.3389/fcimb.2014.00039

130. Sommer F, Bäckhed F. The Gut Microbiota-Masters of Host Development and Physiology. Nat Rev Microbiol (2013) 11(4):227-38. doi: 10.1038/ nrmicro2974

131. Macpherson AJ, Harris NL. Interactions Between Commensal Intestinal Bacteria and the Immune System. Nat Rev Immunol (2004) 4(6):478-85. doi: $10.1038 /$ nri1373 
132. Erny D, Hrabě de Angelis AL, Jaitin D, Wieghofer P, Staszewski O, David E, et al. Host Microbiota Constantly Control Maturation and Function of Microglia in the CNS. Nat Neurosci (2015) 18(7):965-77. doi: 10.1038/ nn. 4030

133. Braniste V, Al-Asmakh M, Kowal C, Anuar F, Abbaspour A, Tóth M, et al. The Gut Microbiota Influences Blood-Brain Barrier Permeability in Mice. Sci Transl Med (2014) 6(263):263ra158-263ra158. doi: 10.1126/ scitranslmed.3009759

Conflict of Interest: The authors declare that the research was conducted in the absence of any commercial or financial relationships that could be construed as a potential conflict of interest.
Publisher's Note: All claims expressed in this article are solely those of the authors and do not necessarily represent those of their affiliated organizations, or those of the publisher, the editors and the reviewers. Any product that may be evaluated in this article, or claim that may be made by its manufacturer, is not guaranteed or endorsed by the publisher.

Copyright $\odot 2021$ Yuan, Lu and Wu. This is an open-access article distributed under the terms of the Creative Commons Attribution License (CC BY). The use, distribution or reproduction in other forums is permitted, provided the original author(s) and the copyright owner(s) are credited and that the original publication in this journal is cited, in accordance with accepted academic practice. No use, distribution or reproduction is permitted which does not comply with these terms. 